\title{
CP-VIOLATION AND UNITARITY TRIANGLE TEST OF THE STANDARD MODEL 1
}

\author{
S. M. Bilenky \\ Joint Institute for Nuclear Research, Dubna, R-141980, Russia
}

\begin{abstract}
Phenomenological issues of the $C P$ violation in the quark sector of the Standard Model are discussed. We consider quark mixing in the $\mathrm{SM}$, standard and Wolfenstein parametrization of the $C K M$ mixing matrix and unitarity triangle. We discuss the phenomenology of the $C P$ violation in $K_{L}^{0}$ and $B_{d}^{0}\left(\bar{B}_{d}^{0}\right)$-decays. The standard unitarity triangle fit of the existing data is discussed. In appendix A we compare the $K^{0} \leftrightarrows \bar{K}^{0}, B_{d, s}^{0} \leftrightarrows \bar{B}_{d, s}^{0}$ etc oscillations with neutrino oscillations. In Appendix B we derive the evolution equation for $M^{0}-\bar{M}^{0}$ system in the Weisskopf-Wigner approximation.
\end{abstract}

\section{Introduction}

Soon after the discovery of the violation of parity $P$ and charge conjugation $C$ in the weak interaction [77] (1957) Landau [2] and Lee and Yang [3] suggested that the Hamiltonian of the weak interaction is invariant under the combined $C P$ transformation. One of the consequence of this suggestion was the theory of the two-component neutrino [2, 3, 4] according to which the neutrino is left-handed (right-handed) particle and antineutrino is right-handed (lefthanded) particle. The helicity of the neutrino was measured in spectacular experiment [5] performed in 1958. This experiment confirmed the theory of the two-component neutrino. It was established that neutrino is left-handed particle.

The confirmation of the theory of the two-component neutrino strengthened belief in the hypothesis of the $C P$ invariance of the Hamiltonian of the weak interaction. All existing data at the end of fifties and beginning of sixties data were in agreement with this hypothesis.

It was a big surprise for the physics community when in the experiment performed by Christenson, Cronin, Fitch and Turlay [6] in 1964 the decay

\footnotetext{
${ }^{1}$ On the basis of the lectures given to the students of SISSA (Trieste) in 2006
} 
$K_{L}^{0} \rightarrow \pi^{+} \pi^{-}$was observed. The observation of this decay was a proof that $C P$ is violated 2

The discovery of the $C P$ violation was announced at the Rochester conference in Dubna. In 1980 Cronin and Fitch were awarded the Nobel Prize for this discovery.

The observed violation of $P$ and $C$ in the $\beta$ decay and other weak decays was large. Discovered by Cronin, Fitch and others effect of the violation of $C P$ was very small. They found that the ratio of the modulus of the amplitudes of the $C P$-forbidden decay $K_{L}^{0} \rightarrow \pi^{+}+\pi^{-}$and the $C P$-allowed decay $K_{S}^{0} \rightarrow \pi^{+}+\pi^{-}$was about $2 \cdot 10^{-3}$.

The first problem was to understand what interaction is responsible for the $C P$ violation in $K_{L}^{0} \rightarrow \pi+\pi$ decays. Many hypothesis were put forward. One of the most viable idea was proposed by Wolfenstein [7]. He noticed that it is possible to explain the observed violation of the $C P$ in decays of $K_{L}^{0}$-meson if we assume that exist a new $|\Delta S|=2$ interaction, which is characterized by a very small effective interaction constant $G_{S W} \simeq 10^{-9} G_{F}\left(G_{F}\right.$ is the Fermi constant). This interaction was called the superweak interaction.

Measurable parameters characterizing violation of $C P$ in $K_{L} \rightarrow \pi+\pi$ decays are $\eta_{+-}$and $\eta_{00}$. These parameters are, correspondingly, ratios of the amplitudes of the decays $K_{L}^{0} \rightarrow \pi^{+}+\pi^{-}$and $K_{S}^{0} \rightarrow \pi^{+}+\pi^{-}$and $K_{L}^{0} \rightarrow \pi^{0}+\pi^{0}$ and $K_{S}^{0} \rightarrow \pi^{0}+\pi^{0}$. If the superweak interaction is responsible for the violation of the $C P$ in $K_{L}^{0} \rightarrow \pi+\pi$ decays in this case

$$
\eta_{+-}=\eta_{00}
$$

It took many years of enormous experimental efforts [8, 9] in order to check the relation (11). It was proved that the relation (11) does not valid. Thus, superweak interaction as a possible source of the $C P$ violation in the neutral kaon decays was excluded by these experiments.

At the time when experiments [8, 9] were completed the Glashow [10], Weinberg 11], Salam [12 Standard Model (SM) was established by numerous experiments. The expected in the SM violation of the relation (1) is very

\footnotetext{
${ }^{2}$ In fact, let us consider decays of short-lived and long-lived kaons $\left(K_{S}^{0}\right.$ and $\left.K_{L}^{0}\right)$ into $\pi^{+}+\pi^{-}$in the rest frame of the kaon. Because spin of the kaon is equal to zero, final pions have equal to zero orbital momentum. Thus, we have $P\left|\pi^{+} \pi^{-}\right\rangle=\left|\pi^{+} \pi^{-}\right\rangle$, $C\left|\pi^{+} \pi^{-}\right\rangle=\left|\pi^{-} \pi^{+}\right\rangle=\left|\pi^{+} \pi^{-}\right\rangle$and $C P\left|\pi^{+} \pi^{-}\right\rangle=\left|\pi^{+} \pi^{-}\right\rangle$. The decay $K_{S} \rightarrow \pi^{+}+\pi^{-}$ is the main decay mode of the short-lived kaon. If $C P$ is conserved, $\left|K_{S}\right\rangle$ is the state with $C P$-parity equal to 1 . The $C P$ parity of the orthogonal state $K_{L}$ must be equal to -1 and hence decay $K_{L} \rightarrow \pi^{+}+\pi^{-}$must be forbidden in the case of the $C P$ conservation.
} 
small (see [13]). The data of the experiments [8, 9] were in agreement with the SM.

In 1973 Kobayashi and Maskawa [14] considered $C P$ violation in the framework of the Standard Model. In the Standard Model violation of the $C P$ is determined by phases in the unitary mixing matrix. In 1973 only two families of leptons and quarks were known. It was demonstrated in [14] that it is impossible to violate $C P$ in this case. It was shown in [14] that in order to explain observed $C P$ violation we need to assume that (at least) six quarks exist. Kobayashi and Maskawa obtained the first parametrization of the mixing matrix in the case of three families. They showed that this matrix is characterized by three mixing angles and one $C P$ phase.

During more than 30 years the investigation of the $C P$ violation was limited by the system of neutral kaons (see book [15]). During last 8 years with the BaBar and Belle experiments at the asymmetric B-factories at the SLAC and KEK a new era in the investigation of the $C P$ violation started (see book [16]). In these experiments numerous effects of the $C P$ violation in different decays of the neutral and charged $B_{d}$-mesons were observed. This allowed to perform the unitarity triangle test of the SM. All existing at present data are in a good agreement with the $S M$ and the assumption that only three families of quarks exist in nature.

In this review we will consider some phenomenological aspects of the problem of the $C P$ violation in the quark sector. In the section 2 we consider the SM Higgs mechanism of the mixing of quarks. In the section 3 we consider in details quark mixing matrix and the $C P$ violation. In the section 4 we derive the standard parametrization of the $C K M$ mixing matrix. In section 5 we discuss the values of the modulus of the elements of the $C K M$ matrix. In section 6 we consider Wolfenstein parametrization of the $C K M$ matrix elements and the unitarity triangle. In the section 7 we obtain eigenstates and eigenvalues of the effective Hamiltonian of $K^{0}-\bar{K}^{0}, B^{0}-\bar{B}^{0}$, etc systems. In the section 8 we consider in details phenomenology of the $C P$ violation in decays of $K_{L}^{0}$. In the section 9 we consider the $C P$ violation in $B^{0}-\bar{B}^{0}$ decays. In the section 10 we present results of the unitarity triangle test of the Standard Model. In the Appendix A we compare of $K^{0} \leftrightarrows \bar{K}^{0}, B^{0} \leftrightarrows \bar{B}^{0}$ etc oscillations with neutrino oscillations. In the Appendix $B$ we derive in the Weisskopf-Wigner approximation the evolution equation for $K^{0}-\bar{K}^{0}$, $B^{0}-\bar{B}^{0}$ etc system.

Last years, in connection with appearance of the B-factories, several books [18, 48], many reviews [16, 20, 21, 22, 23, 24, 25, 13, 26, 27] and hundreds 
papers on the $C P$ violation were published. In these books and reviews many details and many references on original papers can be found.

I tried to discuss here some basic questions and to derive different relations. I hope that this review will be useful for those who start to study this exciting field of physics.

\section{Quark mixing in the Standard Model}

The Standard Model of the electroweak interaction is based on the following principles (see, for example, [28, 29])

1. The local gauge $S U_{L}(2) \times U_{Y}(1)$ symmetry of the Lagrangian of the fields of massless quarks, leptons, gauge vector bosons and scalar Higgs bosons.

2. The spontaneous symmetry breaking. Due to the spontaneous breaking of the local $S U_{L}(2) \times U_{Y}(1)$ symmetry the masses of $W^{ \pm}$and $Z^{0}$ bosons, mass terms of quarks and leptons and mass of the Higgs boson are generated.

3. Unification of the weak and electromagnetic interactions

We will consider the quark sector of the Standard Model. The theory is based on the assumption that the left-handed quark fields are transformed as $S U_{L}(2)$ doublets 3

$$
\psi_{1 L}=\left(\begin{array}{c}
u_{L}^{\prime} \\
d_{L}^{\prime}
\end{array}\right), \quad \psi_{2 L}=\left(\begin{array}{c}
c_{L}^{\prime} \\
s_{L}^{\prime}
\end{array}\right), \quad \psi_{3 L}=\left(\begin{array}{c}
t_{L}^{\prime} \\
b_{L}^{\prime}
\end{array}\right)
$$

and the right-handed fields of quarks $q_{R}^{\prime}(q=u, d, c, s, t, b)$ are the singlets of the group.

The requirements of the local gauge $S U_{L}(2) \times U_{Y}(1)$ invariance fix the Lagrangian of the interaction of quarks and vector bosons in the form of the sum of the charged current (CC), neutral current (NC) and electromagnetic

\footnotetext{
${ }^{3}$ The meaning of primes will be clear later.
} 
(EM) parts:

$$
\begin{aligned}
\mathcal{L}_{I}^{C C} & =-\frac{g}{2 \sqrt{2}} j_{\alpha}^{C C} W^{\alpha}+\text { h.c. }, \\
\mathcal{L}_{I}^{N C} & =-\frac{g}{2 \cos \theta_{W}} j_{\alpha}^{N C} Z^{\alpha}, \\
\mathcal{L}_{I}^{E M} & =-e j_{\alpha}^{E M} A^{\alpha},
\end{aligned}
$$

where

$$
j_{\alpha}^{C C}=2 \sum_{i=1}^{3} \bar{\psi}_{i L} \frac{1}{2}\left(\tau_{1}+i \tau_{2}\right) \gamma_{\alpha} \psi_{i L}=2\left[\bar{u}_{L}^{\prime} \gamma_{\alpha} d_{L}^{\prime}+\bar{c}_{L}^{\prime} \gamma_{\alpha} s_{L}^{\prime}+\bar{t}_{L}^{\prime} \gamma_{\alpha} b_{L}^{\prime}\right]
$$

is the quark charged current,

$$
j_{\alpha}^{N C}=2 \sum_{i=1}^{3} \bar{\psi}_{i L} \frac{1}{2} \tau_{3} \gamma_{\alpha} \psi_{i L}-2 \sin ^{2} \theta_{W} j_{\alpha}^{E M}
$$

is the quark neutral current and

$$
j_{\alpha}^{E M}=\sum_{q=u, d, c, \ldots} e_{q} \bar{q}^{\prime} \gamma_{\alpha} q^{\prime}
$$

is the electromagnetic current. Here $W^{\alpha}$ is the field of $W^{ \pm}$bosons, $Z^{\alpha}$ is the field of $Z^{0}$ bosons, $A^{\alpha}$ is the electromagnetic field, $g$ is the electroweak constant, $\theta_{W}$ is the weak angle, $e_{q}=2 / 3,-1 / 3$ are the quark charges.

In the total Lagrangian of the Standard Model enter the following $S U_{L}(2) \times$ $U_{Y}(1)$ invariant Lagrangians of the Yukawa interaction of quarks and Higgs fields

$$
\mathcal{L}_{Y}^{\text {down }}=-\frac{\sqrt{2}}{v} \sum_{i=1,2,3} \bar{\psi}_{i L=d, s, b} M_{i q}^{\text {down }} q_{R}^{\prime} \phi+\text { h.c. }
$$

and

$$
\mathcal{L}_{Y}^{\mathrm{up}}=-\frac{\sqrt{2}}{v} \sum_{i=1,2,3} \bar{\psi}_{i L} M_{i q}^{\mathrm{up}} q_{R}^{\prime} \tilde{\phi} .,+ \text { h.c. }
$$

Here $M^{\text {down }}$ and $M^{\text {up }}$ are complex $3 \times 3$ matrices, $\phi=\left(\begin{array}{c}\phi_{+} \\ \phi_{0}\end{array}\right)$ is the Higgs doublet, $\tilde{\phi}=i \tau_{2} \phi^{*}$ and $v$ is the constant (vacuum expectation value of the Higgs field). 
If we choose

$$
\phi(x)=\left(\begin{array}{c}
0 \\
\frac{v+\chi(x)}{\sqrt{2}}
\end{array}\right)
$$

where $\chi(x)$ is the field of neutral Higgs bosons, the symmetry will be spontaneously broken. For the mass terms of up and down quarks we obtain the following expressions

$$
\mathcal{L}_{m}^{\text {up }}=-\overline{U^{\prime}}{ }_{L} M^{\text {up }} U_{R}^{\prime}+\text { h.c. }, \quad \mathcal{L}_{\mathrm{m}}^{\text {down }}=-\overline{\mathrm{D}^{\prime}}{ }_{\mathrm{L}} \mathrm{M}^{\text {down }} \mathrm{D}_{\mathrm{R}}^{\prime}+\text { h.c. },
$$

where

$$
U_{L, R}^{\prime}=\left(\begin{array}{c}
u_{L, R}^{\prime} \\
c_{L, R}^{\prime} \\
t_{L, R}^{\prime}
\end{array}\right), \quad D_{L, R}^{\prime}=\left(\begin{array}{c}
d_{L, R}^{\prime} \\
s_{L, R}^{\prime} \\
b_{L, R}^{\prime}
\end{array}\right)
$$

The complex matrices $M^{\text {up }}$ and $M^{\text {down }}$ can be diagonalized by the biunitary transformations

$$
M^{\text {up }}=V_{L}^{\text {up }} m^{\text {up }} V_{R}^{\text {up } \dagger}, \quad M^{\text {down }}=V_{L}^{\text {down }} m^{\text {down }} V_{R}^{\text {down } \dagger} .
$$

Here $V_{L, R}^{\text {up }}$ and $V_{L, R}^{\text {down }}$ are unitary matrices and $m^{\text {up }}$ and $m^{\text {down }}$ are diagonal matrices with positive diagonal elements.

From (10) and (12) we find

$$
\mathcal{L}_{m}^{\text {up }}=-\bar{U} m^{\text {up }} U, \quad \mathcal{L}_{m}^{\text {down }}=-\bar{D} m^{\text {down }} D .
$$

Here

$$
\begin{gathered}
U=U_{L}+U_{R}=\left(\begin{array}{c}
u \\
c \\
t
\end{array}\right), \quad D=D_{L}+D_{R}=\left(\begin{array}{l}
d \\
s \\
b
\end{array}\right), \\
m^{\text {up }}=\left(\begin{array}{ccc}
m_{u} & 0 & 0 \\
0 & m_{c} & 0 \\
0 & 0 & m_{t}
\end{array}\right), \quad m^{\text {down }}=\left(\begin{array}{ccc}
m_{d} & 0 & 0 \\
0 & m_{s} & 0 \\
0 & 0 & m_{b}
\end{array}\right)
\end{gathered}
$$

and

$$
U_{L, R}=V_{L, R}^{\mathrm{up} \dagger} U_{L, R}^{\prime}, \quad D_{L, R}=V_{L, R}^{\mathrm{down} \dagger} D_{L, R}^{\prime}
$$


From (13), (14) and (16) we obtain the standard mass terms for up and down quarks

$$
\mathcal{L}_{m}^{\text {up }}(x)=-\sum_{q=u, c, t} m_{q} \bar{q}(x) q(x), \quad \mathcal{L}_{m}^{\text {down }}(x)=-\sum_{q=d, s, b} m_{q} \bar{q}(x) q(x)
$$

Thus, $q(x)$ is the field of the $q$-quarks with the mass $m_{q}(q=u, d, c, s, t, b)$. The left-handed and right-handed fields of quarks with definite masses and primed quark fields, which have definite transformation properties, are connected by the unitary transformations (16).

Let us consider now the charged current of the quarks. From (44) and (16) we find

$j_{\alpha}^{C C}=2 \bar{U}_{L}^{\prime} \gamma_{\alpha} D_{L}^{\prime}=2 \bar{U}_{L} \gamma_{\alpha} V D_{L}=2\left[\bar{u}_{L} \gamma_{\alpha} d_{L}^{\text {mix }}+\bar{c}_{L} \gamma_{\alpha} s_{L}^{\text {mix }}+\bar{t}_{L} \gamma_{\alpha} b_{L}^{\text {mix }}\right]$.

Here

$$
V=\left(V_{L}^{\text {up }}\right)^{\dagger} V_{L}^{\text {down }}
$$

and

$$
d_{L}^{\text {mix }}=\sum_{d_{1}=d, s, b} V_{u d_{1}} d_{1 L}, \quad s_{L}^{\text {mix }}=\sum_{d_{1}=d, s, b} V_{c d_{1}} d_{1 L}, \quad b_{L}^{\text {mix }}=\sum_{d_{1}=d, s, b} V_{t d_{1}} d_{1 L} .
$$

From (19) it follows that $V$ is unitary matrix 4

$$
V^{\dagger} V=1 .
$$

From (18) and (20) we conclude that fields of down quarks enter into $C C$ of the SM in the form of the "mixed " combinations $d_{L}^{\text {mix }}, s_{L}^{\text {mix }}, b_{L}^{\text {mix }}$. The unitary $3 \times 3$ mixing matrix $V$ is called Cabibbo [30]-Kobayashi-Maskawa [14] $(C K M)$ mixing matrix. We will see later that the violation of the $C P$ invariance is determined in the $S M$ by the matrix $V$.

Let us consider now the electromagnetic current. From (6) we have

$$
j_{\alpha}^{E M}=\frac{2}{3}\left(\bar{U}_{L}^{\prime} \gamma_{\alpha} U_{L}^{\prime}+\bar{U}_{R}^{\prime} \gamma_{\alpha} U_{R}^{\prime}\right)-\frac{1}{3}\left(\bar{D}_{L}^{\prime} \gamma_{\alpha} D_{L}^{\prime}+\bar{D}_{R}^{\prime} \gamma_{\alpha} D_{R}^{\prime}\right) .
$$

Taking into account the unitarity of the matrices $V_{L, R}^{\text {up }}$ and $V_{L, R}^{\text {down }}$, we find $j_{\alpha}^{E M}=\frac{2}{3}\left(\bar{U}_{L} \gamma_{\alpha} U_{L}+\bar{U}_{R} \gamma_{\alpha} U_{R}\right)-\frac{1}{3}\left(\bar{D}_{L} \gamma_{\alpha} D_{L}+\bar{D}_{R} \gamma_{\alpha} D_{R}\right)=\sum_{q=u, d, c, \ldots} e_{q} \bar{q} \gamma_{\alpha} q$,

\footnotetext{
${ }^{4}$ We assume that there are no additional heavy families of quarks.
} 
where $e_{u, c, t}=\frac{2}{3}$ and $e_{d, s, b}=-\frac{1}{3}$. Thus, we come to the standard expression for the electromagnetic current which is diagonal in the quark flavors.

Let us consider the neutral current. We have

$$
\begin{aligned}
j_{\alpha}^{N C} & =2 \sum_{i}^{3} \bar{\psi}_{i L} \frac{1}{2} \tau_{3} \gamma_{\alpha} \psi_{i L}-2 \sin ^{2} \theta_{W} j_{\alpha}^{E M} \\
& =\bar{U}_{L}^{\prime} \gamma_{\alpha} U_{L}^{\prime}-\bar{D}_{L}^{\prime} \gamma_{\alpha} D_{L}^{\prime}-2 \sin ^{2} \theta_{W} j_{\alpha}^{E M} \\
& =\sum_{u_{1}=u, c, t} \bar{u}_{1 L} \gamma_{\alpha} u_{1 L}-\sum_{d_{1}=d, s, b} \bar{d}_{1 L} \gamma_{\alpha} d_{1 L}-2 \sin ^{2} \theta_{W} j_{\alpha}^{E M} .
\end{aligned}
$$

Thus, the neutral current of the SM is also diagonal in the quark flavors. Only the charged current changes flavor of the quarks ( $s \rightarrow u+W^{-}$etc). We will show later that the electromagnetic and NC interactions of the SM automatically conserve $C P$. The $C P$ invariance can be violated only by the flavor-changing $C C$ interaction.

\section{Mixing matrix}

We will consider here general properties of the unitary mixing matrix $V$. Let us calculate first the number of the angles and phases which characterize the unitary mixing matrix $V$ in the general $n \times n$ case.

The unitary matrix $V$ can be presented in the form $V=e^{i H}$, where $H$ is the hermitian matrix. Such matrix is characterized by $n$ (diagonal elements) $+2\left(\frac{n^{2}-n}{2}\right)$ (nondiagonal elements) $=n^{2}$ real parameters.

The number of the angles which characterize $n \times n$ unitary matrix coincides with the number of parameters which characterize $n \times n$ orthogonal matrix $O\left(O^{T} O=1\right)$. Such matrix can be presented in the form $O=e^{A}$, where $A^{T}=-A$. The antisymmetric matrix $A$ is characterized

by $\frac{n(n-1)}{2}$ (nondiagonal elements) real parameters. Thus, the number of the angles which characterize the unitary matrix is equal to

$$
n_{\text {angles }}=\frac{n(n-1)}{2}
$$

Other parameters of the matrix $V$ are phases. The number of the phases is equal to

$$
n_{\text {phases }}=n^{2}-\frac{n(n-1)}{2}=\frac{n(n+1)}{2} \text {. }
$$


The number of physical phases, which characterize mixing matrix, is significantly smaller than $n_{\text {phases }}$.

The mixing matrix enter into $\mathrm{CC}$ together with the quark fields:

$$
j_{\alpha}^{C C}=2 \sum_{u_{1}=u, c, t, t d_{1}=d, s, b} \bar{u}_{1 L} \gamma_{\alpha} V_{u_{1} d_{1}} d_{1 L} .
$$

The free Lagrangian of quark fields is invariant under the transformation

$$
q(x) \rightarrow e^{i \alpha_{q}} q(x), \quad q=u, d, \ldots
$$

where $\alpha_{q}$ is an arbitrary constant phase. quark fields are We will take this fact into account in the calculation of the number of physical phases in the mixing matrix $V$.

The unitary matrix can be presented in the form

$$
V=S^{\dagger}(\alpha) \tilde{V} S(\beta),
$$

where $S(\alpha)$ and $S(\beta)$ are diagonal phase matrices $\left(S_{u_{1} u_{2}}(\alpha)=\delta_{u_{1} u_{2}} e^{i \alpha_{u_{1}}} ; S_{d_{1} d_{2}}(\beta)=\right.$ $\left.\delta_{d_{1} d_{2}} e^{i \beta_{d_{1}}}\right)$ and $\tilde{V}$ is an unitary matrix. There are $2(n-1)+1$ independent phases $\alpha_{u_{1}}$ and $\beta_{d_{1}}$.

The phase factors $e^{i \alpha_{u_{1}}}$ and $e^{i \beta_{d_{1}}}$ can be included into quark fields. Thus, the number of measurable, physical phases which characterize unitary mixing matrix $\tilde{V}$ is equal to

$$
n_{\mathrm{phases}}^{\mathrm{phys}}=\frac{n(n+1)}{2}-(2 n-1)=\frac{(n-1)(n-2)}{2} .
$$

Let us obtain now the constraints on the mixing matrix which follow from the requirements of the $C P$ invariance of the $C C$ interaction. For the $C C$ Lagrangian we have

$$
\begin{aligned}
\mathcal{L}_{I}^{C C}(x) & =-\frac{g}{\sqrt{2}} \sum_{u_{1}=u, c, t d_{1}=d, s, b} \bar{u}_{1 L}(x) \gamma^{\alpha} V_{u_{1} d_{1}} d_{1 L}(x) W_{\alpha}(x) \\
& -\frac{g}{\sqrt{2}} \sum_{u_{1}=u, c, t d_{1}=d, s, b} \bar{d}_{1 L}(x) \gamma^{\alpha} V_{u_{1} d_{1}}^{*} u_{1 L}(x) W_{\alpha}^{\dagger}(x),
\end{aligned}
$$

where $V$ is the $3 \times 3$ unitary $C K M$ mixing matrix (we suppressed tilde).

\footnotetext{
${ }^{5}$ We must take into account that only difference of common phases of $S(\beta)$ and $S(\alpha)$ enters into (29)
} 
The $C P$ is conserved if Lagrangian satisfies the following condition

$$
V_{C P} \mathcal{L}_{I}^{C C}(x) V_{C P}^{-1}=\mathcal{L}_{I}^{C C}\left(x^{\prime}\right),
$$

where $V_{C P}$ is the operator of the $C P$ conjugation and $x^{\prime}=\left(x^{0},-\vec{x}\right)$.

For the left-handed quark field $q_{L}(x)$ we have

$$
V_{C P} q_{L}(x) V_{C P}^{-1}=e^{-2 i \alpha_{q}} \gamma^{0} C \bar{q}_{L}^{T}\left(x^{\prime}\right) .
$$

Here $\alpha_{q}$ is an arbitrary phase and $C$ is the matrix of the charge conjugation, which satisfies the relations

$$
C \gamma_{\alpha}^{T} C^{-1}=-\gamma_{\alpha}, \quad C^{T}=-C .
$$

Taking into account that phases of quark fields are arbitrary, we can include phase factor $e^{i \alpha_{q}}$ into the field $q(x)$. We obtain in this case

$$
V_{C P} q_{L}(x) V_{C P}^{-1}=\gamma^{0} C \bar{q}_{L}^{T}\left(x^{\prime}\right) .
$$

From (34) from (35) we also have

$$
V_{C P} \bar{q}_{L}(x) V_{C P}^{-1}=-q_{L}^{T}\left(x^{\prime}\right) C^{-1} \gamma^{0} .
$$

Let us consider now the current $\bar{u}_{1 L}(x) \gamma_{\alpha} d_{1 L}(x)$. From (34), (35) and (36) we find

$$
\begin{aligned}
V_{C P} \bar{u}_{1 L}(x) \gamma_{\alpha} d_{1 L}(x) V_{C P}^{-1} & =-u_{1 L}^{T}\left(x^{\prime}\right) C^{-1} \gamma^{0} \gamma_{\alpha} \gamma^{0} C \bar{d}_{1 L}\left(x^{\prime}\right) \\
& =-\delta_{\alpha} \bar{d}_{1 L}\left(x^{\prime}\right) \gamma_{\alpha} u_{1 L}\left(x^{\prime}\right) .
\end{aligned}
$$

Here $\delta=(1,-1,-1,-1)$ is the sign factor. Notice that in the relation (37) we took into account anticommutator properties of the fermion fields.

Under the $C P$ transformation the field of the vector $W^{ \pm}$bosons is transformed as follows

$$
V_{C P} W_{\alpha}(x) V_{C P}^{-1}=-e^{-2 i \beta_{W}} \delta_{\alpha} W_{\alpha}^{\dagger}\left(x^{\prime}\right),
$$

where $\beta_{W}$ is an arbitrary phase. Taking into account that phase of the nonhermitian $W_{\alpha}(x)$ field is arbitrary, we can include phase factor $e^{i \beta_{W}}$ into the $W$ field. In this case we have

$$
V_{C P} W_{\alpha}(x) V_{C P}^{-1}=-\delta_{\alpha} W_{\alpha}^{\dagger}\left(x^{\prime}\right) .
$$


With the help of (31), (37) and (39) we find

$$
\begin{aligned}
V_{C P} \mathcal{L}_{I}^{C C}(x) V_{C P}^{-1} & =-\frac{g}{\sqrt{2}} \sum_{u_{1}, d_{1}} \bar{d}_{1 L}\left(x^{\prime}\right) \gamma^{\alpha} V_{u_{1} d_{1}} u_{1 L}\left(x^{\prime}\right) W_{\alpha}^{\dagger}\left(x^{\prime}\right) \\
& -\frac{g}{\sqrt{2}} \sum_{u_{1}, d_{1}} \bar{u}_{1 L}\left(x^{\prime}\right) \gamma^{\alpha} V_{u_{1} d_{1}}^{*} d_{1 L}\left(x^{\prime}\right) W_{\alpha}\left(x^{\prime}\right) .
\end{aligned}
$$

From (31), (32) and (40) we conclude that in the case of the $C P$ invariance the $C K M$ mixing matrix $V$ is real:

$$
V_{u_{1} d_{1}}=V_{u_{1} d_{1}}^{*}
$$

We will comment now this condition. The first term of the CC Lagrangian (31) is responsible for the flavor-changing transition

$$
d_{1} \rightarrow u_{1}+W^{-}, \quad d_{1}=d, s, b, \quad u_{1}=u, c, t .
$$

Amplitude of this transition is equal to $V_{u_{1} d_{1}}$. The second term of the Lagrangian (31) is responsible for the $C P$-conjugated transition

$$
\bar{d}_{1} \rightarrow \bar{u}_{1}+W^{+}, \quad \bar{d}_{1}=\bar{d}, \bar{s}, \bar{b}, \quad \bar{u}_{1}=\bar{u}, \bar{c}, \bar{t} .
$$

Because the Lagrangian is hermitian the amplitude of the transition (43) is equal to $V_{u_{1} d_{1}}^{*}$. If the $C P$ invariance holds the amplitude of transition (42) is equal to the amplitudes of $C P$-conjugated transition (43).

As we have shown the number of the physical phases in the $C K M$ mixing matrix is given by (30). For $n=2$ the mixing matrix is real. Thus, for two families of quarks the unitarity of the mixing matrix assures invariance of the Lagrangian of interaction of the quarks and $W$-bosons under $C P$ transformation 6

For $n=3$ number of measurable phases in the mixing matrix is equal to one 7 It follows from (41) that in the case of the $C P$ invariance this phase must be equal to zero.

\footnotetext{
${ }^{6}$ In order to explain in the framework of the SM observed violation of the $C P$ invariance we need to assume that (at least) three families of quarks exist in nature. This was original argument of Kobayashi and Maskawa [14 in favor of the existence of the third family of quarks. When this argument was presented only two families of quarks were known.

${ }^{7}$ The minimal number of families at which the CC Lagrangian of the SM can violate $C P$ is equal to three. This minimal number is equal to the number of SM families of quarks and leptons which exist in nature. In fact, it was established by the experiments on the measurement of the width of the decay $Z \rightarrow \nu+\bar{\nu}$ that the number of flavor neutrinos is equal to three. (see 32]). This means that the number of the lepton families is equal to three. For the SM to be renormalizable the number of the quark families must be also equal to three.
} 
We have considered the CC part of the SM interaction Lagrangian. Let us discuss now the neutral current and electromagnetic interactions. From (34), (35) and (36) for the left-handed current we have

$$
V_{C P} \bar{q}_{L}(x) \gamma_{\alpha} q_{L}(x) V_{C P}^{-1}=-\delta_{\alpha} \bar{q}_{L}\left(x^{\prime}\right) \gamma_{\alpha} q_{L}\left(x^{\prime}\right) .
$$

Analogously, for right-handed current we obtain

$$
V_{C P} \bar{q}_{R}(x) \gamma_{\alpha} q_{R}(x) V_{C P}^{-1}=-\delta_{\alpha} \bar{q}_{R}\left(x^{\prime}\right) \gamma_{\alpha} q_{R}\left(x^{\prime}\right) .
$$

Taking into account that

$$
V_{C P} Z^{\alpha}(x) V_{C P}^{-1}=-\delta_{\alpha} Z^{\alpha}\left(x^{\prime}\right), \quad V_{C P} A^{\alpha}(x) V_{C P}^{-1}=-\delta_{\alpha} A^{\alpha}\left(x^{\prime}\right)
$$

from (3), (23) and (24) we find

$$
V_{C P} \mathcal{L}_{I}^{N C}(x) V_{C P}^{-1}=\mathcal{L}_{I}^{N C}\left(x^{\prime}\right), \quad V_{C P} \mathcal{L}_{I}^{E M}(x) V_{C P}^{-1}=\mathcal{L}_{I}^{E M}\left(x^{\prime}\right) .
$$

Thus, the SM Lagrangians of the NC and electromagnetic interactions are automatically invariant under $C P$ transformation. This is connected with the fact that the electromagnetic and neutral current interactions of the SM are diagonal in the quark flavors.

We have chosen $C P$ phase factors of quark and $W$ fields equal to one and determined $C P$ transformations by the relations (35) and (39). In this case $C K M$ matrix is characterized by three angles and one phase responsible for the violation of the $C P$ invariance. It is of interest to characterize $C P$ violation in a rephrasing-invariant way [31].

Let us consider quantities

$$
Q_{u_{1} u_{2}}^{d_{1} d_{2}}=V_{u_{1} d_{1}} V_{u_{2} d_{2}} V_{u_{1} d_{2}}^{*} V_{u_{2} d_{1}}^{*}
$$

invariant under phase transformation

$$
V_{u_{i} d_{k}} \rightarrow e^{-i \alpha_{u_{i}}} V_{u_{i} d_{k}} e^{i \beta_{d_{k}}},
$$

where $\alpha_{u_{i}}$ and $\beta_{d_{k}}$ are arbitrary phases. It is evident that

$$
\left(Q_{u_{1} u_{2}}^{d_{1} d_{2}}\right)^{*}=Q_{u_{1} u_{2}}^{d_{2} d_{1}}=Q_{u_{2} u_{1}}^{d_{1} d_{2}}
$$

If we determine the $C P$ conjugation by the relations (33) and (38) with arbitrary $C P$ phases of the quark and $W$ fields from the $C P$ invariance of the $\mathrm{CC}$ Lagrangian we find

$$
e^{2 i \alpha_{u_{1}}} V_{u_{1} d_{1}} e^{-2 i \alpha_{d_{1}}} e^{-2 i \beta_{W}}=V_{u_{1} d_{1}}^{*} .
$$


It follows from (48) and (51) that in the case of the $C P$ invariance the quantities $Q_{u_{1} u_{2}}^{d_{1} d_{2}}$ are real:

$$
Q_{u_{1} u_{2}}^{d_{1} d_{2}}=V_{u_{1} d_{1}}^{*} V_{u_{2} d_{2}}^{*} V_{u_{1} d_{2}} V_{u_{2} d_{1}}=\left(Q_{u_{1} u_{2}}^{d_{1} d_{2}}\right)^{*}
$$

Let us introduce the quantities

$$
J_{u_{1} u_{2}}^{d_{1} d_{2}}=\operatorname{Im} \mathrm{Q}_{\mathrm{u}_{1} \mathrm{u}_{2}}^{\mathrm{d}_{1} \mathrm{~d}_{2}}
$$

In the case of the $C P$ invariance we have

$$
J_{u_{1} u_{2}}^{d_{1} d_{2}}=0
$$

In the general case of the $C P$ violation from (50) we obtain the following relations

$$
J_{u_{1} u_{2}}^{d_{1} d_{2}}=-J_{u_{1} u_{2}}^{d_{2} d_{1}}, \quad J_{u_{1} u_{2}}^{d_{1} d_{2}}=-J_{u_{2} u_{1}}^{d_{1} d_{2}} .
$$

Thus, $J_{u_{1} u_{2}}^{d_{1} d_{2}} \neq 0$ only if $d_{1} \neq d_{2}$ and $u_{1} \neq u_{2}$.

Further, from the unitarity of the mixing matrix we find

$$
\sum_{d_{1}} Q_{u_{1} u_{2}}^{d_{1} d_{2}}=\delta_{u_{1} u_{2}} V_{u_{2} d_{2}} V_{u_{1} d_{2}}^{*}, \quad \sum_{u_{1}} Q_{u_{1} u_{2}}^{d_{1} d_{2}}=\delta_{d_{1} d_{2}} V_{u_{2} d_{2}} V_{u_{2} d_{1}}^{*}
$$

From these relations we have

$$
\sum_{d_{1}} J_{u_{1} u_{2}}^{d_{1} d_{2}}=0, \quad \sum_{u_{1}} J_{u_{1} u_{2}}^{d_{1} d_{2}}=0
$$

Let us consider first the simplest case of two families. We have in this case

$$
J_{u c}^{d s}=0
$$

This result corresponds to the absence of the physical phases in the mixing matrix for $n=2$.

We will consider now the case of three families. From the first relation (57) we have

$$
J_{u_{1} u_{2}}^{s d}+J_{u_{1} u_{2}}^{b d}=0, J_{u_{1} u_{2}}^{d s}+J_{u_{1} u_{2}}^{b s}=0, J_{u_{1} u_{2}}^{d b}+J_{u_{1} u_{2}}^{s b}=0 .
$$

It follows from (59) and (55) that the following cycling relations hold

$$
J_{u_{1} u_{2}}^{d s}=J_{u_{1} u_{2}}^{s b}=J_{u_{1} u_{2}}^{b d}
$$


From the second relation (57) we obtain following equations

$$
J_{c u}^{d_{1} d_{2}}+J_{t u}^{d_{1} d_{2}}=0, J_{u c}^{d_{1} d_{2}}+J_{t c}^{d_{1} d_{2}}=0, J_{u t}^{d_{1} d_{2}}+J_{c t}^{s d}=0 .
$$

From these relations and (155) we find

$$
J_{u c}^{d_{1} d_{2}}=J_{c t}^{d_{1} d_{2}}=J_{t u}^{d_{1} d_{2}} .
$$

From (60) and (62) we obtain the following relations

$$
J_{u c}^{d s}=J_{c t}^{d s}=J_{t u}^{d s}=J_{u c}^{s b}=J_{u c}^{b d}=\ldots=J .
$$

Other nonzero $J_{u_{1} u_{2}}^{d_{1} d_{2}}$ differ from $J$ by $\operatorname{sign}\left(J_{u c}^{b s}=-J\right.$ etc). Thus, in the case of three families exist only one independent rephrasing invariant quantity. This result is determined by the fact that for $n=3$ there is only one physical phase in the mixing matrix. The quantity $J$ is called Jarskog invariant.

\section{Standard parametrization of the $C K M$ mix- ing matrix}

Several parameterizations of the unitary $C K M$ mixing matrix $V$ were proposed in literature. We will obtain here the so called standard parametrization [32] which is based on the three Euler rotations.

Let us consider three orthogonal and normalized vectors

$$
|d\rangle, \quad|s\rangle \text { and }|\mathrm{b}\rangle \text {. }
$$

In order to obtain three general "mixed" vectors we will perform the three Euler rotations. The first rotation will be performed at the angle $\theta_{12}$ around the vector $|b\rangle$. New orthogonal and normalized vectors are

$$
\begin{array}{lcc}
|d\rangle^{\prime}= & c_{12}|d\rangle+s_{12}|s\rangle \\
|s\rangle^{\prime}= & -s_{12}|d\rangle+c_{12}|s\rangle \\
|b\rangle^{\prime}= & |b\rangle,
\end{array}
$$

where $c_{12}=\cos \theta_{12}$ and $s_{12}=\sin \theta_{12}$. In the matrix form (65) can be written as follows

$$
|D\rangle^{\prime}=V^{\prime}|D\rangle
$$


Here

$$
|D\rangle^{\prime}=\left(\begin{array}{c}
|d\rangle^{\prime} \\
|s\rangle^{\prime} \\
|b\rangle^{\prime}
\end{array}\right), \quad|D\rangle=\left(\begin{array}{c}
|d\rangle \\
|s\rangle \\
|b\rangle
\end{array}\right)
$$

and

$$
V^{\prime}=\left(\begin{array}{ccc}
c_{12} & s_{12} & 0 \\
-s_{12} & c_{12} & 0 \\
0 & 0 & 1
\end{array}\right)
$$

Let us perform now the second rotation at the angle $\theta_{13}$ around the vector $|s\rangle^{\prime}$. At this step we will introduce the $C P$ phase $\delta$, connected with the rotation of the vector of the third family $|b\rangle$. We will obtain the following three orthogonal vectors:

$$
\begin{array}{lc}
|d\rangle^{\prime \prime}= & c_{13}|d\rangle^{\prime}+s_{13} e^{-i \delta}|b\rangle^{\prime} \\
|s\rangle^{\prime \prime}= & \quad|s\rangle^{\prime} \\
|b\rangle^{\prime \prime}= & -s_{13} e^{i \delta}|d\rangle^{\prime}+c_{13}|b\rangle^{\prime} .
\end{array}
$$

In the matrix form we have

$$
|D\rangle^{\prime \prime}=V^{\prime \prime}|D\rangle^{\prime}
$$

Here

$$
V^{\prime \prime}=\left(\begin{array}{ccc}
c_{13} & 0 & s_{13} e^{-i \delta} \\
0 & 1 & 0 \\
-s_{13} e^{i \delta} & 0 & c_{13}
\end{array}\right)
$$

Finally, let us perform rotation around the vector $|d\rangle^{\prime \prime}$ at the angle $\theta_{23}$. New orthogonal vectors are

$$
\begin{array}{lc}
|d\rangle^{\prime \prime \prime}= & |d\rangle^{\prime \prime} \\
|s\rangle^{\prime \prime \prime}= & c_{23}|s\rangle^{\prime \prime}+s_{13}|b\rangle^{\prime \prime} \\
|b\rangle^{\prime \prime \prime}= & -s_{23}|s\rangle^{\prime \prime}+c_{23}|b\rangle^{\prime \prime}
\end{array}
$$

We have

$$
\left|D^{\prime \prime \prime}\right\rangle=V^{\prime \prime \prime}\left|D^{\prime \prime}\right\rangle
$$


Here

$$
V^{\prime \prime \prime}=\left(\begin{array}{ccc}
1 & 0 & 0 \\
0 & c_{23} & s_{23} \\
0 & -s_{23} & c_{23}
\end{array}\right)
$$

From (166), (70) and(173) we find

$$
\left|D^{\prime \prime \prime}\right\rangle=V|D\rangle
$$

where

$$
V=V^{\prime \prime \prime} V^{\prime \prime} V^{\prime}
$$

It is obvious that $V$ is the unitary matrix.

Thus, the general $3 \times 3$ unitary mixing matrix has the form

$$
V=\left(\begin{array}{ccc}
1 & 0 & 0 \\
0 & c_{23} & s_{23} \\
0 & -s_{23} & c_{23}
\end{array}\right)\left(\begin{array}{ccc}
c_{13} & 0 & s_{13} e^{-i \delta} \\
0 & 1 & 0 \\
-s_{13} e^{i \delta} & 0 & c_{13}
\end{array}\right)\left(\begin{array}{ccc}
c_{12} & s_{12} & 0 \\
-s_{12} & c_{12} & 0 \\
0 & 0 & 1
\end{array}\right)
$$

From (177) we find

$$
V=\left(\begin{array}{ccc}
c_{13} c_{12} & c_{13} s_{12} & s_{13} e^{-i \delta} \\
-c_{23} s_{12}-s_{23} c_{12} s_{13} e^{i \delta} & c_{23} c_{12}-s_{23} s_{12} s_{13} e^{i \delta} & c_{13} s_{23} \\
s_{23} s_{12}-c_{23} c_{12} s_{13} e^{i \delta} & -s_{23} c_{12}-c_{23} s_{12} s_{13} e^{i \delta} & c_{13} c_{23}
\end{array}\right) .
$$

In the standard parametrization the $3 \times 3$ mixing matrix is characterized by three Euler angles $\theta_{12}, \theta_{23}$ and $\theta_{13}$ and one phase $\delta$. We have seen before that in the case of the $C P$ conservation $V^{*}=V$. Thus, in this case $\delta=0$.

Let us calculate in the standard parametrization of the $C K M$ mixing matrix the invariant $J$ given by (63). From (78) we have

$$
J=c_{12} c_{23} c_{13}^{2} s_{12} s_{23} s_{13} \sin \delta .
$$

As we have seen in the previous section in the case of the $C P$ conservation the Jarlskog invariant $J$ is equal to zero. It follows from experimental data that all mixing angles are different from zero. (see below). The rephrase invariant condition of the $C P$ conservation has the form: $\sin \delta=0$. 


\section{Modulus of the elements of $C K M$ matrix}

The values of the modulus of the $C K M$ matrix elements were determined from the data of different experiments (see [33]).

The highest accuracy was reached in the measurement of the element $\left|V_{u d}\right|$. There are three sources of information about this element: i) The superallowed $0^{+} \rightarrow 0^{+} \beta$-decay of nuclei. ii) The neutron decay. iii) The $\beta$-decay of pion $\pi^{+} \rightarrow \pi^{0} e^{+} \nu_{e}$.

Only vector current gives contribution to the matrix element of the $0^{+} \rightarrow$ $0^{+} \beta$-transition. From the isotopic invariance and the $C V C$ follows that matrix element of $0^{+} \rightarrow 0^{+}$transition between components of isotopic triplet is given by

$$
\left|\left\langle p^{\prime}\left|V_{\alpha}\right| p\right\rangle\right|=N\left|V_{u d}\right| \sqrt{2}\left(p+p^{\prime}\right)_{\alpha}
$$

where $p$ and $p^{\prime}$ are momenta of initial and final nuclei and $N$ is the normalization factor. The nuclear Coulomb effects and radiative corrections, which violate this relation, must be taken into account. From the most precise measurements of the $f t$ values of nine nuclei the following average value was obtained [34, 35]

$$
\left|V_{u d}\right|=0.97377 \pm 0.00027
$$

It is necessary to notice, however, that the $Q$-value of ${ }^{46} \mathrm{~V}$ was recently remeasured [35]. The new value leads to an increase of the $f$-factor which gives $2.7 \sigma$ decrease of the value of $\left|V_{u d}\right|$ with respect to the average value (81).

The element $\left|V_{u d}\right|$ can be determined also from data of the experiments on the measurements of the lifetime of the neutron $\tau_{n}$ and from the ratio of axial and vector constants $g_{A}$. The constant $g_{A}$ can be obtained from the data of the experiments on the measurement of the asymmetry of electrons in decay of polarized neutrons. From the world averages values of $\tau_{n}$ and $g_{A}$ 32

$$
\tau_{n}=885.7 \pm 0.8 \mathrm{sec}, \quad \mathrm{g}_{\mathrm{A}}=-1.2695 \pm 0.0029
$$

for the element $\left|V_{u d}\right|$ it was found the value [37]

$$
\left|V_{u d}\right|=0.9746 \pm 0.0004 \pm 0.0018 \pm 0.0002 .
$$

Here the first (second) error is due to the error of $\tau_{n}\left(g_{A}\right)$ and the third error is due to the uncertainty in the calculations of radiative corrections. As it is seen from (83) the dominant uncertainty is due to the error of the constant $g_{A}$. 
Finally, the value of the element $\left|V_{u d}\right|$ was obtained from the measurement of the branching ratio of the decay $\pi^{+} \rightarrow \pi^{0} e^{+} \nu_{e}$. Only vector $C C$ current gives contribution to the hadronic matrix element of this process. From the $C V C$ and isotopic invariance it follows that matrix element of the hadronic vector current is given by the relation (80). The problem of the calculation of the radiative corrections is much more simpler in the pion case than in the nuclear case. However, the branching ratio of the pion $\beta$-decay is very small $\left(B\left(\pi^{+} \rightarrow \pi^{0} e^{+} \nu_{e}\right) \simeq 10^{-8}\right)$. As a result, the accuracy of the determination of the element $\left|V_{u d}\right|$ from the measurement of this branching ratio is much worse than from the measurement of the $f t$ values of the nuclear $0^{+} \rightarrow 0^{+}$ $\beta$-decays. In [36] it was found the value

$$
\left|V_{u d}\right|=0.9728 \pm 0.0030 \text {. }
$$

The value of the element $\left|V_{u s}\right|$ was obtained from the measurement of the widths of the decays $K_{L} \rightarrow \pi^{ \pm} l^{\mp} \nu_{l}(l=e, \mu)$ and $K^{+} \rightarrow \pi^{0} e^{+} \nu_{e}$. Only CC vector current gives contribution to the hadronic part of the matrix elements of these decays. The matrix element is characterized by the two form factors and has the form

$$
\left\langle p^{\prime}\left|V_{\alpha}\right| p\right\rangle=N V_{u d}\left(f_{+}\left(Q^{2}\right)\left(p+p^{\prime}\right)_{\alpha}+f_{-}\left(Q^{2}\right)\left(p-p^{\prime}\right)_{\alpha}\right) .
$$

Here $p$ and $p^{\prime}$ are momenta of kaon and pion, $Q^{2}=-\left(p^{\prime}-p\right)^{2}$ and $N$ is the standard normalization factor. Taking into account the results of the measurements of the form factors $f_{ \pm}\left(Q^{2}\right)$ and recent measurements of the branching ratios of the decays $K_{L} \rightarrow \pi e \nu$ and $K_{L} \rightarrow \pi \mu \nu$ [38, 39, 40] for the element $\left|V_{u s}\right|$ the following value was found [37]

$$
\left|V_{u s}\right|=0.2257 \pm 0.0021
$$

This result was obtained with the chiral perturbation value [41] $f_{+}(0)=$ $0.961 \pm 0.008$ was used.

The value of the parameter $\left|V_{u s}\right|$ can be also obtained from the measurement of the widths of the decays $K^{+} \rightarrow \mu^{+} \nu_{\mu}$ and $\pi^{+} \rightarrow \mu^{+} \nu_{\mu}$. Using for the ratio of the decay constants the value

$$
\frac{f_{K}}{f_{\pi}}=1.198_{-0.005}^{+0.016} \pm 0.003,
$$

which was obtained in the lattice calculations [42], for the matrix element $\left|V_{u s}\right|$ it was found [37]

$$
\left|V_{u s}\right|=0.2245_{-0.0031}^{+0.0012}
$$


The value of the element $\left|V_{u s}\right|$ can be also inferred from the analysis of data on the investigation of the hyperon decays. From these data it was found [43]

$$
\left|V_{u s}\right|=0.2250 \pm 0.0027
$$

Finally, an information about the value of the parameter $\left|V_{u s}\right|$ can be obtained from the data of the experiments on the investigation of the decays $\tau^{ \pm} \rightarrow$ $\nu_{\tau}+$ hadrons $(\mathrm{S}= \pm 1)$. From these data the following value of the matrix element $\left|V_{u s}\right|$ was found [4]

$$
\left|V_{u s}\right|=0.2208 \pm 0.0034
$$

Thus, the values of the element $\left|V_{u s}\right|$, determined from the different experimental data and with different theoretical inputs, are compatible.

From the unitarity of the $C K M$ matrix $V$ we have

$$
\left|V_{u d}\right|^{2}+\left|V_{u s}\right|^{2}+\left|V_{u b}\right|^{2}=1
$$

The last term gives negligible contribution to this relation (see later). From (83) and (86) it was found [37]

$$
\left|V_{u d}\right|^{2}+\left|V_{u s}\right|^{2}+\left|V_{u b}\right|^{2}=0.9992 \pm 0.0005 \pm 0.0009
$$

where the first error is due to the error of $\left|V_{u d}\right|$ and the second one is due to the error of $\left|V_{u s}\right|$. Thus, the values (83) and (86) of the parameters $\left|V_{u d}\right|$ and $\left|V_{u s}\right|$ saturate the unitarity relation (91).

The element $\left|V_{c d}\right|$ can be determined from the data on the production of the muon pairs in the processes of interaction of $\nu_{\mu}$ and $\bar{\nu}_{\mu}$ with nucleons 8 From these data was found [33]

$$
\left|V_{c d}\right|=0.230 \pm 0.011
$$

The element $\left|V_{c d}\right|$ can be also obtained from the data on the study of the decays $D \rightarrow \pi l \nu_{l}$ if the corresponding form factors are known. Using lattice calculations of the form factors [45] it was found [46]

$$
\left|V_{c d}\right|=0.213 \pm 0.008 \pm 0.021
$$

where the dominant error is the theoretical one.

\footnotetext{
${ }^{8}$ One muon is produced in a process of interaction of neutrino (antineutrino) with nucleon and another in decay of produced charmed particle.
} 
The value of the element $\left|V_{c s}\right|$ was determined from the data on the investigation of the decays $D \rightarrow K l \nu_{l}$. Using the lattice calculations of the form factors [45] it was found the value [46]

$$
\left|V_{c s}\right|=0.957 \pm 0.017 \pm 0.093
$$

where the second (theoretical) error is the largest one.

An model independent information about the element $\left|V_{c s}\right|$ can be obtained from the data on the study of the decay $W^{+} \rightarrow c+\bar{s}$. From the LEP data it was found the value [47]

$$
\left|V_{c s}\right|=0.94_{-0.26}^{+0.32} \pm 0.13 \text {. }
$$

The value of the element $\left|V_{c b}\right|$ was determined from the data on the investigation of the semileptonic inclusive decays $\bar{B} \rightarrow X_{c} l \bar{\nu}_{l}$ and exclusive $\bar{B} \rightarrow D\left(D^{*}\right) l \bar{\nu}_{l}$ decays. Analysis of the inclusive data is based on the operator product expansion theory [48, 49]. From the LEP and $B$-factories data it was found the following average value [50]

$$
\left|V_{c b}\right|=(41.7 \pm 0.7) \cdot 10^{-3}
$$

Analysis of the exclusive data is based on the heavy quark effective theory [51, 52]. The average value

$$
\left|V_{c b}\right|=(40.9 \pm 1.8) \cdot 10^{-3}
$$

which was found from analysis of the exclusive data [50] is compatible with (97).

The value of the element $\left|V_{u b}\right|$ can be obtained from the study of semileptonic inclusive decay

$$
\bar{B} \rightarrow X_{u} l \bar{\nu}_{l}
$$

and exclusive decay

$$
\bar{B} \rightarrow \pi l \bar{\nu}_{l}
$$

The suppression of the background from the $C K M$ enhanced inclusive decay $\bar{B} \rightarrow X_{c} l \bar{\nu}_{l}$ is the main problem in the investigation of the decay (99). The following average value of $\left|V_{u b}\right|$ was obtained from different inclusive measurements [50]

$$
\left|V_{u b}\right|=(4.40 \pm 0.20 \pm 0.27) \cdot 10^{-3}(\text { inclusive }) .
$$


In the exclusive decay (100) both final charged particles are detected. This leads to the better suppression of the background than in the inclusive case. However, the branching ratio of the exclusive decay (which is known at present with the accuracy $\sim 8 \%$ ) is much smaller than the branching ratio of the inclusive decay. The hadronic matrix element of the process (100) is given by

$$
\left\langle p^{\prime}\left|V_{\alpha}\right| p\right\rangle=N V_{u b}\left(f_{+}\left(q^{2}\right)\left(p+p^{\prime}-\frac{m_{B}^{2}-m_{\pi}^{2}}{q^{2}}\right) q_{\alpha}+f_{0}\left(q^{2}\right) \frac{m_{B}^{2}-m_{\pi}^{2}}{q^{2}} q_{\alpha}\right),
$$

where $q=p-p^{\prime}$ and $f_{+}\left(q^{2}\right), f_{0}\left(q^{2}\right)$ are the form factors.

The calculation of the form factors $f_{+}\left(q^{2}\right)$ and $f_{0}\left(q^{2}\right)$ is the main problem in the determination of $\left|V_{u b}\right|$ from the exclusive data. Using lattice calculations [58, 54] the following value was found [50]

$$
\left|V_{u b}\right|=\left(3.84_{-0.49}^{+0.67}\right) \cdot 10^{-3}(\text { exclusive })
$$

This value is compatible with (101). From (101) and (103) the following weighted average of the matrix element $\left|V_{u b}\right|$ was obtained [50]

$$
\left|V_{u b}\right|=(4.31 \pm 0.39) \cdot 10^{-3} \text { (exclusive) }
$$

The element $\left|V_{t d}\right|$ can be determined from the measurement of the mass difference of $B_{d}^{0}$ mesons. The major contribution to the box diagram which determine mass differences $\Delta m_{q}(q=d, s)$ gives the virtual $t$-quark. We have (see, for example, [13])

$$
\Delta m_{q}=\frac{G_{F}^{2}}{6 \pi^{2}} m_{B_{q}} m_{W}^{2}\left(f_{B_{q}}^{2} \hat{B}_{B_{q}}\right) \eta_{B} S_{0}\left(x_{t}\right)\left|V_{t b} V_{t q}^{*}\right|^{2} .
$$

Here $f_{B_{q}}$ is the decay constant and $\hat{B}_{B_{q}}$ is so called B-factor. The factor $\eta_{B}$ is due to short distance $Q C D$ corrections $\left(\eta_{B}=0.55 \pm 0.01\right)$ and $S_{0}\left(x_{t}\right)$ is known function of $x_{t}=\frac{m_{t}^{2}}{m_{t}^{2}}$.

For the mass difference $\Delta m_{d}$ the following value was obtained [55]

$$
\Delta m_{d}=(0.507 \pm 0.004) \mathrm{ps}^{-1} .
$$

Assuming $\left|V_{t b}\right|=1$ and taking into account the lattice result [56, 57]

$$
f_{B_{d}} \sqrt{\hat{B}_{B_{d}}}=(244 \pm 11 \pm 24) \mathrm{MeV}
$$


for the element $\left|V_{t d}\right|$ it was found the value [58]

$$
\left|V_{t d}\right|=(7.4 \pm 0.8) 10^{-3}
$$

Recently the mass difference of $B_{s}^{0}$ mesons was measured. Using the CDF value [59]

$$
\Delta m_{s}=\left(17.31_{-0.18}^{+0.33} \pm 0.07\right) \mathrm{ps}^{-1}
$$

and the lattice result

$$
\frac{f_{B_{s}} \sqrt{\hat{B}_{B_{s}}}}{f_{B_{d}} \sqrt{\hat{B}_{B_{d}}}}=1.21 \pm 0.04_{-0.01}^{+0.04}
$$

it was obtained [59]

$$
\frac{\left|V_{t d}\right|}{\left|V_{t s}\right|}=0.208_{-0.006}^{+0.008}
$$

The value of the element $\left|V_{t s}\right|$ can be found from the unitarity relation $V_{c b} V_{c s}^{*}+V_{t b} V_{t s}^{*}+V_{u b} V_{u s}^{*}=0$. It was obtained [33]

$$
\left|V_{t s}\right|=(40.6 \pm 2.7) 10^{-3} \text {. }
$$

Finally, an information about the element $\left|V_{t b}\right|$ can be inferred from the measurement of the ratio $\frac{B(t \rightarrow W b)}{\sum_{q=d, s, b} B(t \rightarrow W q)}=\left|V_{t b}\right|^{2}$. From the Fermilab data [60, 61] it was found the following $95 \%$ CL lower bound [33]

$$
\left|V_{t b}\right|>0.78 \text {. }
$$

\section{Wolfenstein parameters. Unitarity triangle}

From the values of the modulus of elements of the $C K M$ matrix, which we discussed in the previous section, it follows that quark mixing angles are small and exist a hierarchy of mixing between different families. In fact, in the standard parametrization of the $C K M$ matrix we have

$$
V_{u d}=c_{13} c_{12}, \quad V_{u s}=c_{13} s_{12}, \quad V_{c b}=c_{13} s_{23}, \quad V_{u b}=s_{13} e^{-i \delta} .
$$

From these relations we find

$$
s_{12}=\frac{\left|V_{u s}\right|}{\sqrt{\left|V_{u d}\right|^{2}+\left|V_{u s}\right|^{2}}}, \quad s_{23}=\frac{\left|V_{c b}\right|}{\sqrt{\left|V_{u d}\right|^{2}+\left|V_{u s}\right|^{2}}}, \quad s_{13}=\left|V_{u b}\right| .
$$


From (81), (86), (197), (101) and (115) for the parameters $s_{i k}$ we find

$$
s_{12} \sim 2 \cdot 10^{-1}, \quad s_{23} \sim 4 \cdot 10^{-2}, \quad s_{13} \sim 4 \cdot 10^{-3} .
$$

Let us introduce the parameter

$$
\lambda=s_{12}
$$

We have

$$
s_{23} \simeq \lambda^{2}, \quad s_{13} \simeq \frac{1}{2} \lambda^{3} .
$$

Thus, exist a hierarchy of angles of the mixing between different quark families. The strength of the coupling between the families is determined by the degree of the parameter $\lambda$.

Wolfenstein [62] proposed a parametrization of the mixing matrix which take into account this hierarchy. Instead of $s_{12}, s_{23}$ and $s_{13} e^{-i \delta}$ he introduced four real parameters $\lambda, A, \rho$ and $\eta$ by the following relations

$$
s_{12}=\lambda, s_{23}=A \lambda^{2}, s_{13} e^{-i \delta}=A \lambda^{3}(\rho-i \eta) .
$$

Let us develop elements of the $C K M$ matrix over the small parameter $\lambda$. Keeping terms of the order of $\lambda^{5}$ for the $C K M$ mixing matrix $V$ we have

$$
V=\left(\begin{array}{ccc}
1-\frac{1}{2} \lambda^{2}-\frac{1}{8} \lambda^{4} & \lambda & A \lambda^{3}(\rho-i \eta) \\
-\lambda+\frac{1}{2} A^{2} \lambda^{5}(1-2(\rho+i \eta)) & 1-\frac{1}{2} \lambda^{2}-\frac{1}{8} \lambda^{4}\left(1+4 A^{2}\right) & A \lambda^{2} \\
A \lambda^{3}\left(1-\left(1-\frac{1}{2} \lambda^{2}\right)(\rho+i \eta)\right) & -A \lambda^{2}+\frac{1}{2} A \lambda^{4}(1-2(\rho+i \eta)) & 1-\frac{1}{2} A^{2} \lambda^{4}
\end{array}\right)
$$

We will obtain now the so called unitarity triangle relation. This relation follows from the condition of the unitarity of the mixing matrix

$$
V^{\dagger} V=1
$$

For the three families of the quarks from (121) we have

$$
\sum_{u_{1}=u, c, t} V_{u_{1} d_{1}}^{*} V_{u_{1} d_{2}}=\delta_{d_{1} d_{2}}
$$

From (122) we obtain the following relations

$$
\sum_{u_{1}=u, c, t}\left|V_{u_{1} d}\right|^{2}=1, \quad \sum_{u_{1}=u, c, t}\left|V_{u_{1} s}\right|^{2}=1, \quad \sum_{u_{1}=u, c, t}\left|V_{u_{1} b}\right|^{2}=1
$$


and

$$
\sum_{u_{1}=u, c, t} V_{u_{1} d} V_{u_{1} s}^{*}=0, \quad \sum_{u_{1}=u, c, t} V_{u_{1} s} V_{q b}^{*}=0, \quad \sum_{u_{1}=u, c, t} V_{u_{1} d} V_{u_{1} b}^{*}=0 .
$$

Let us consider the relations (124). In the first relation the first and the second terms are of the order $\lambda$ and the third one is of the order $\lambda^{5}$. Thus, in this relation the main contribution give terms which connect only two families (the first and the second). In the second relation (124) the first term is of the order $\lambda^{4}$ and the second and the third terms are of the order $\lambda^{2}$. In this relation the main contribution also give terms which connect only two families (the second and third). The only relation in which all terms are of the same $\left(\lambda^{3}\right)$ order is the third relation (124). It has the form

$$
V_{u d} V_{u b}^{*}+V_{c d} V_{c b}^{*}+V_{t d} V_{t b}^{*}=0 .
$$

Let us now expand different terms of (125) over the powers of the small parameter $\lambda$. We have 63]

$$
V_{u d} V_{u b}^{*}=c_{13} c_{12} s_{13} e^{i \delta}=A \lambda^{3}(\bar{\rho}+i \bar{\eta})+O\left(\lambda^{7}\right)
$$

where

$$
\bar{\rho}=\left(1-\frac{1}{2} \lambda^{2}\right) \rho, \quad \bar{\eta}=\left(1-\frac{1}{2} \lambda^{2}\right) \eta
$$

For the second term of the relation (125) we find

$$
V_{c d} V_{c b}^{*}=\left(-s_{12} c_{23}-c_{12} s_{23} s_{13} e^{i \delta}\right) c_{13} s_{23}=-A \lambda^{3}+O\left(\lambda^{7}\right) .
$$

Finally, for the third term of (125) we obtain

$$
V_{t d} V_{t b}^{*}=\left(s_{23} s_{12}-c_{23} c_{12} s_{13} e^{i \delta}\right) c_{13} c_{23}=\simeq A \lambda^{3}(1-(\bar{\rho}+i \bar{\eta}))+O\left(\lambda^{7}\right) .
$$

We see from the relations (126), (128) and (129) that up to small terms of the order of $\lambda^{7}$ all terms in (125) are proportional to $A \lambda^{3}$.

Let us rewrite the relation (125) in the form

$$
\frac{V_{u d} V_{u b}^{*}}{\left(-V_{c d} V_{c b}^{*}\right)}+\frac{V_{t d} V_{t b}^{*}}{\left(-V_{c d} V_{c b}^{*}\right)}=1
$$

We have 9

$$
\frac{V_{u d} V_{u b}^{*}}{\left(-V_{c d} V_{c b}^{*}\right)}=\bar{\rho}+i \bar{\eta}=\sqrt{\bar{\rho}^{2}+\bar{\eta}^{2}} e^{i \gamma}
$$

\footnotetext{
${ }^{9}$ It is obvious that the ratios of the products of the $C K M$ matrix elements in (130) are invariant under phase transformation (49).
} 
and

$$
\frac{V_{t d} V_{t b}^{*}}{\left(-V_{c d} V_{c b}^{*}\right)}=1-(\bar{\rho}+i \bar{\eta})=\sqrt{(1-\bar{\rho})^{2}+\bar{\eta}^{2}} e^{-i \beta} .
$$

Thus, the unitarity relation (130) takes the form

$$
(\bar{\rho}+i \bar{\eta})+(1-(\bar{\rho}+i \bar{\eta}))=1 \text {. }
$$

This relation can be presented as a triangle in the complex $(\bar{\rho}, \bar{\eta})$ plane (see Fig.1). It is called the unitarity triangle.

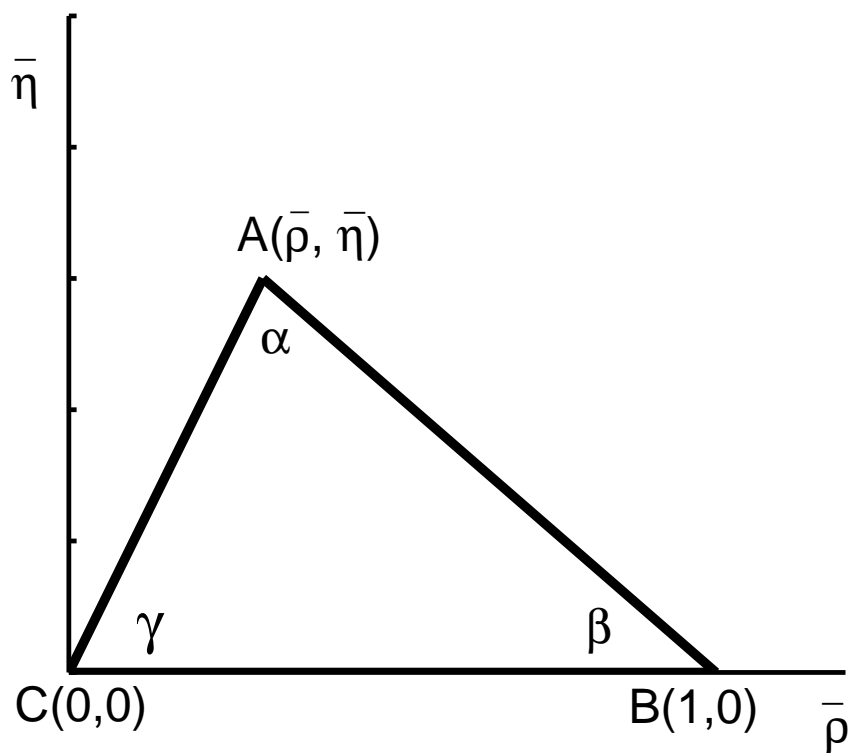

Figure 1: The unitarity triangle. The angles $\alpha \equiv \phi_{2}, \beta \equiv \phi_{1}, \gamma \equiv \phi_{3}$ are shown

From (132) and (133) for the angles $\gamma$ and $\beta$ we have 10

$$
\gamma=\arg \left(-\frac{V_{u d} V_{u b}^{*}}{V_{c d} V_{c b}^{*}}\right), \quad \beta=\arg \left(-\frac{V_{c d} V_{c b}^{*}}{V_{t d} V_{t b}^{*}}\right) .
$$

\footnotetext{
${ }^{10}$ Other notations for the angles of the unitarity triangle, which are often used in literature, are: $\phi_{1} \equiv \beta ; \phi_{2} \equiv \alpha ; \phi_{3} \equiv \gamma$.
} 
From (78) and (131) it follows that the angle $\gamma$ coincides with the $C K M$ angle $\delta$. For the angle $\alpha$ we find

$$
\alpha=\pi-\beta-\gamma=\arg \left(-\frac{V_{t d} V_{t b}^{*}}{V_{u d} V_{u b}^{*}}\right) .
$$

The square of the unitarity triangle is equal to

$$
\tilde{S}=\frac{1}{2} 1 \cdot \bar{\eta}
$$

From (131) we find

$$
-\frac{V_{u d} V_{c b} V_{u b}^{*} V_{c d}^{*}}{\left|V_{c d}\right|^{2}\left|V_{c b}\right|^{2}}=\bar{\rho}+i \bar{\eta} .
$$

From this relation we have

$$
\bar{\eta}=\frac{J}{\left|V_{c d}\right|^{2}\left|V_{c b}\right|^{2}},
$$

where $J$ is the Jarskog invariant (63). Thus, the square of the unitarity triangle is given by

$$
\tilde{S}=\frac{1}{2} \frac{J}{\left|V_{c d}\right|^{2}\left|V_{c b}\right|^{2}} .
$$

For the square of the unitarity triangle, given by the relation (130), we have [31]

$$
S=\frac{1}{2} J
$$

The relations (124) are conditions of orthogonality of the columns of the matrix $V$. Additional three relations can be obtained from the conditions of orthogonality of the lines of the matrix $V$. We can see easily that the only relation in which all terms are of the same $\lambda^{3}$ order is the condition of the orthogonality of the first and the third lines:

$$
\sum_{d_{1}=d, s, b} V_{u d_{1}} V_{t d_{1}}^{*}=0
$$

This relation after expansion of different terms over the powers of the parameter $\lambda$ also takes the form of the relation (133). 


\section{Eigenstates and eigenvalues of the effective Hamiltonian of the $M^{0}-\bar{M}^{0}$ system}

We will obtain here eigenstates and eigenvalues of the effective $2 \times 2$ nonhermitian Hamiltonian $\mathcal{H}$ of the $M^{0}-\bar{M}^{0}$ system $\left(M^{0}=K^{0}, B_{d, s}^{0}, \ldots\right)$ which we derived in the Appendix B. We have

$$
\mathcal{H} a_{H, L}=\mu_{H, L} a_{H, L}
$$

Here

$$
\mu_{H, L}=m_{H, L}-\frac{i}{2} \Gamma_{H, L}
$$

and

$$
a_{H, L}=\left(\begin{array}{c}
a_{H, L}(1) \\
a_{H, L}(2)
\end{array}\right)
$$

If the wave function at the initial time $t=0$ is equal to $a_{H, L}$, at $t \geq 0$ we have

$$
a_{H, L}(t)=e^{-i m_{H, L} t-\frac{1}{2} \Gamma_{H, L} t} a_{S, L} .
$$

Thus, $m_{H, L}$ and $\Gamma_{S, L}$ are masses and total decay widths of $M_{H, L}^{0}$-bosons, particles which are described by the functions $a_{H, L}$. We will use the index $H$ for the heavier particle and the index $L$ for the lighter particle. Thus, we have $m_{H}>m_{L}$. For the vectors of the states of $M_{H, L}^{0}$ we have

$$
\left|M_{H, L}^{0}\right\rangle=\sum_{\alpha=1,2} a_{H, L}(\alpha)|\alpha\rangle
$$

where $|1\rangle \equiv\left|M^{0}\right\rangle$ and $|2\rangle \equiv\left|\bar{M}^{0}\right\rangle$ are states of $M^{0}$ and $\bar{M}^{0}$ particles (in the rest frame system).

Assuming the CPT invariance of the Hamiltonian, we can present the effective Hamiltonian of the $M^{0}-\bar{M}^{0}$ system in the form

$$
\mathcal{H}=\mathcal{H}_{11}+\mathcal{H}^{\mathrm{nd}}
$$

where

$$
\mathcal{H}^{\text {nd }}=\left(\begin{array}{cc}
0 & \mathcal{H}_{12} \\
\mathcal{H}_{21} & 0
\end{array}\right)
$$


From (146) and (147) we have

$$
\mathcal{H}^{\text {nd }} a_{H, L}=\kappa_{H, L} a_{H, L} .
$$

Here

$$
\kappa_{H, L}=\mu_{H, L}-\mathcal{H}_{11}
$$

For the eigenvalues $\kappa_{S, L}$ of the Hamiltonian $\mathcal{H}^{\text {nd }}$ we obviously have

$$
\kappa_{H, L}=\mp \sqrt{\mathcal{H}_{12} \mathcal{H}_{21}}
$$

Further from (148) and (150) we find that $a_{H, L}(2)$ and $a_{H, L}(1)$ are connected by the relation

$$
a_{H, L}(2)=\mp \sqrt{\frac{\mathcal{H}_{21}}{\mathcal{H}_{12}}} a_{H, L}(1),
$$

where $a_{H, L}(1)$ is an arbitrary constant.

The equation (141) have the following solutions

$$
a_{H, L}=\left(\begin{array}{c}
1 \\
\mp \sqrt{\frac{\mathcal{H}_{21}}{\mathcal{H}_{12}}}
\end{array}\right) a_{H, L}(1)
$$

with

$$
\mu_{H, L}=\mathcal{H}_{11} \mp \sqrt{\mathcal{H}_{12} \mathcal{H}_{21}} .
$$

Three physical complex parameters $\mu_{H, L}$ (masses and total decay width of $\left.M_{H, L}^{0}\right)$ and parameter $\sqrt{\frac{\mathcal{H}_{21}}{\mathcal{H}_{12}}}$, which characterize mixing of $M^{0}$ and $\bar{M}^{0}$, correspond to three complex matrix elements of the matrix $\mathcal{H}\left(\mathcal{H}_{11}, \mathcal{H}_{12}\right.$ and $\left.\mathcal{H}_{21}\right)$.

Let us choose

$$
a_{H, L}(1)=\frac{\sqrt{\mathcal{H}_{12}}}{\sqrt{\left|\mathcal{H}_{12}\right|+\left|\mathcal{H}_{21}\right|}}=p
$$

We have

$$
a_{H, L}=\left(\begin{array}{c}
p \\
\mp q
\end{array}\right)
$$

where

$$
q=\frac{\sqrt{\mathcal{H}_{21}}}{\sqrt{\left|\mathcal{H}_{12}\right|+\left|\mathcal{H}_{21}\right|}}
$$


With this choice we have

$$
|p|^{2}+|q|^{2}=1 \text { and }\left(\mathrm{a}_{\mathrm{H}, \mathrm{L}}^{\dagger} \mathrm{a}_{\mathrm{H}, \mathrm{L}}\right)=1 .
$$

The states of $M_{H, L}^{0}$ are given by the following relations

$$
\left|M_{H}^{0}\right\rangle=p\left|M^{0}\right\rangle-q\left|\bar{M}^{0}\right\rangle, \quad\left|M_{L}^{0}\right\rangle=p\left|M^{0}\right\rangle+q\left|\bar{M}^{0}\right\rangle .
$$

If $C P$ is conserved in this case $\mathcal{H}_{21}=\mathcal{H}_{12}$ and $q=p$. For the eigenstates of the Hamiltonian we have

$$
\left|M_{2,1}^{0}\right\rangle=\frac{1}{\sqrt{2}}\left(\left|M^{0}\right\rangle \mp\left|\bar{M}^{0}\right\rangle\right) .
$$

Let us make the following remark. We have chosen phases of the states $\left|M^{0}\right\rangle$ and $\left|\bar{M}^{0}\right\rangle$ in such a way that (see Appendix B)

$$
C P\left|M^{0}\right\rangle=\left|\bar{M}^{0}\right\rangle
$$

The states $\left|M^{0}\right\rangle$ and $\left|\bar{M}^{0}\right\rangle$ are eigenstates of the Hamiltonians of the strong and electromagnetic interactions. These interactions conserve quark flavors. This means that it is impossible to distinguish states $\left|M^{0}\right\rangle$ and $\left|\bar{M}^{0}\right\rangle$ from the states

$$
\left|M^{0}\right\rangle^{\prime}=e^{i \alpha}\left|M^{0}\right\rangle, \quad\left|\bar{M}^{0}\right\rangle^{\prime}=e^{-i \alpha}\left|\bar{M}^{0}\right\rangle,
$$

where $\alpha$ is an arbitrary phase.

If for the states of $M^{0}$ and $\bar{M}^{0}$ we will use $\left|M^{0}\right\rangle^{\prime}$ and $\left|\bar{M}^{0}\right\rangle^{\prime}$ in this case we have

$$
p^{\prime}=e^{-i \alpha} p, \quad q^{\prime}=e^{i \alpha} q .
$$

The states of $M_{H, L}^{0}$ are invariant under the change of the basis. In fact, we have

$$
\left|M_{H, L}^{0}\right\rangle^{\prime}=p^{\prime}\left|M^{0}\right\rangle^{\prime} \mp q^{\prime}\left|\bar{M}^{0}\right\rangle^{\prime}=p\left|M^{0}\right\rangle \mp q\left|\bar{M}^{0}\right\rangle=\left|M_{H, L}^{0}\right\rangle .
$$

\section{$8 C P$ violation in the decays of $K_{L}^{0}$-meson}

The observation of the decay $K_{L}^{0} \rightarrow \pi^{+} \pi^{-}$marked the discovery of the $C P$ violation [6]. During more than 30 years the study of decays of neutral kaons was the only source of the information about the $C P$ violation. In this section we will consider in some details effects of the $C P$ violation in decays of $K_{L}^{0}$-mesons (see [64]).

The branching ratios of main decay modes of $K_{S, L}^{0}$-mesons are presented in the Table I [32] 
Table I

Branching ratios for main decay channels of $K_{S, L}^{0}$-mesons.

\begin{tabular}{|cccc|}
\hline$K_{S^{-}}^{0}$ decay channels & Branching ratio & $K_{L^{0} \text {-decay channels }}$ & Branching ratio \\
\hline$K_{S}^{0} \rightarrow \pi^{+} \pi^{-}$ & $68.95 \pm 0.14 \%$ & $K_{L}^{0} \rightarrow \pi^{+} e^{-} \nu_{e}$ & $38.8 \pm 0.27 \%$ \\
$K_{S}^{0} \rightarrow \pi^{0} \pi^{0}$ & $31.05 \pm 0.14 \%$ & $K_{L} \rightarrow \pi^{+} \mu^{-} \nu_{\mu}$ & $27.19 \pm 0.25 \%$ \\
$K_{S}^{0} \rightarrow \pi^{+} \pi^{-} \pi^{0}$ & $(3.2 \pm 1.2) 10^{-7}$ & $K_{L}^{0} \rightarrow 3 \pi^{0}$ & $21.05 \pm 0.23 \%$ \\
- & - & $K_{L}^{0} \rightarrow \pi^{+} \pi^{-} \pi^{0}$ & $12.59 \pm 0.19 \%$ \\
\hline
\end{tabular}

As it is seen from the Table I $K_{S}^{0}$-meson decays mainly into two pions and $K_{L}^{0}$-meson decays mainly into three particles: three pions and pion, lepton, neutrino. Because the phase-space factor in the case of the decay into two particles is much larger than in the case of the decay into three particles, the time of life of $K_{L}$ is much larger than the time of life of $K_{S}$ [32]:

$$
\tau_{L}=\frac{1}{\Gamma_{L}}=(5.18 \pm 0.04) \cdot 10^{-8} \mathrm{sec}, \tau_{\mathrm{S}}=\frac{1}{\Gamma_{\mathrm{S}}}=(0.8953 \pm 0.006) \cdot 10^{-10} \mathrm{sec}
$$

For the ratio of the widths of $K_{S}^{0}$ and $K_{L}^{0}$ we have

$$
\frac{\Gamma_{S}}{\Gamma_{L}} \simeq 580
$$

For the masses of $K_{S}^{0}$ and $K_{L}^{0}$ it was found the value [32]

$$
m_{S, L}=(497.648 \pm 0.022) \mathrm{MeV}
$$

For the difference of the masses of $K_{L}^{0}$ and $K_{S}^{0}$ mesons the following value was found 32 ]

$\Delta m=m_{L}-m_{S}=(0.5992 \pm 0.0010) \cdot 10^{10} \hbar s^{-1}=(3.483 \pm 0.006) \cdot 10^{-12} \mathrm{MeV}$

Let us notice the following approximate empirical relation

$$
\frac{1}{2} \Gamma_{S} \simeq \Delta m
$$

We will consider the $C P$ forbidden decays

$$
K_{L}^{0} \rightarrow \pi^{+}+\pi^{-} \quad \text { and } \quad \mathrm{K}_{\mathrm{L}}^{0} \rightarrow \pi^{0}+\pi^{0} .
$$


For the states of $K_{L, S}$-mesons we have

$$
\left|K_{L}^{0}\right\rangle=p\left|K^{0}\right\rangle-q\left|\bar{K}^{0}\right\rangle, \quad\left|K_{S}^{0}\right\rangle=p\left|K^{0}\right\rangle+q\left|\bar{K}^{0}\right\rangle
$$

where the parameters $p$ and $q$ are given by (154) and (156), respectively.

The $\left|K_{L, S}^{0}\right\rangle$ states can be presented in another form. Let us introduce the complex parameter 11

$$
\bar{\epsilon}=\frac{1-\frac{q}{p}}{1+\frac{q}{p}}=\frac{p-q}{p+q}
$$

From (170) and (171) for the normalized $\left|K_{L, S}^{0}\right\rangle$ states we find

$$
\left|K_{L, S}^{0}\right\rangle=\frac{1}{\sqrt{2\left(1+|\bar{\epsilon}|^{2}\right)}}\left[(1+\bar{\epsilon})\left|K^{0}\right\rangle \mp(1-\bar{\epsilon})\left|\bar{K}^{0}\right\rangle\right] .
$$

If $C P$ is conserved, in this case $\bar{\epsilon}=0$ and for the states of the long-lived and the short-lived kaons we have

$$
\left|K_{2,1}^{0}\right\rangle=\frac{1}{\sqrt{2}}\left(\left|K^{0}\right\rangle \mp\left|\bar{K}^{0}\right\rangle\right) .
$$

The states $\left|K_{2,1}^{0}\right\rangle$ are eigenstates of the operator of the $C P$ conjugation:

$$
C P\left|K_{2,1}^{0}\right\rangle=\mp\left|K_{2,1}^{0}\right\rangle \text {. }
$$

The states $\left|K_{L, S}^{0}\right\rangle$ can be presented in the form

$$
\left|K_{L}^{0}\right\rangle=\frac{1}{\sqrt{\left(1+|\bar{\epsilon}|^{2}\right)}}\left(\left|K_{2}^{0}\right\rangle+\bar{\epsilon}\left|\bar{K}_{1}^{0}\right\rangle\right), \quad\left|K_{S}^{0}\right\rangle=\frac{1}{\sqrt{\left(1+|\bar{\epsilon}|^{2}\right)}}\left(\left|K_{1}^{0}\right\rangle+\bar{\epsilon}\left|\bar{K}_{2}^{0}\right\rangle\right)
$$

Let us introduce the measurable parameters

$$
\eta_{+-}=\frac{\left\langle\pi^{+} \pi^{-}|T| K_{L}^{0}\right\rangle}{\left\langle\pi^{+} \pi^{-}|T| K_{S}^{0}\right\rangle}, \quad \eta_{00}=\frac{\left\langle\pi^{0} \pi^{0}|T| K_{L}^{0}\right\rangle}{\left\langle\pi \pi|T| K_{S}^{0}\right\rangle},
$$

which characterize the $C P$ violation in the decays (169) 12 In (176) $T$-matrix is connected with the $S$-matrix by the relation $S=1+i T$.

\footnotetext{
${ }^{11}$ The parameter $\bar{\epsilon}$ characterizes $C P$ violation in the $K_{L, S}^{0}$ states. Let us stress, however, that $\bar{\epsilon}$ depends on arbitrary phases of the $\left|K^{0}\right\rangle$ and $\left|\bar{K}^{0}\right\rangle$ states.

12 In fact, the states $\left|\pi^{+} \pi^{-}\right\rangle$and $\left|\pi^{0} \pi^{0}\right\rangle$ are eigenstates of the operator of the $C P$ conjugation with eigenvalues equal to 1 . If $C P$ is conserved, the state of the long-lived kaon is $\left|K_{2}^{0}\right\rangle$, which is eigenstate of $C P$ with eigenvalue equal to -1 . Thus, in the case of the $C P$ conservation $\eta_{+-}=\eta_{00}=0$.
} 
The complex parameters $\eta_{+-}$and $\eta_{00}$ can be presented in the form

$$
\eta_{+-}=\left|\eta_{+-}\right| e^{i \phi_{+-}}, \quad \eta_{00}=\left|\eta_{00}\right| e^{i \phi_{00}} .
$$

From analysis of the experimental data it was found [32]

$$
\begin{aligned}
\left|\eta_{+-}\right| & =(2.288 \pm 0.014) \cdot 10^{-3},\left|\eta_{+-}\right|=(2.276 \pm 0.014) \cdot 10^{-3} \\
\phi_{+-} & =(43.52 \pm 0.06)^{\circ}, \phi_{+-}=(43.50 \pm 0.06)^{\circ} .
\end{aligned}
$$

The spin of the kaon is equal to zero, Thus, in the $K_{L, S^{-}}^{0}$ decays two pions are produced in the $S$-state. Total isotopic spin $I$ of two pions takes the values $0,1,2$. However, from the Bose-Einstein statistics it follows that state with $I=1$ is forbidden. Hence, the states of two pions, produced in the decays of $K_{L, S}$ are superpositions of states with the total isotopic spin equal to 0 and 2. We have

$$
\begin{aligned}
\left|\pi^{+} \pi^{-}\right\rangle & =\sqrt{\frac{2}{3}}|0\rangle+\sqrt{\frac{1}{3}}|2\rangle \\
\left|\pi^{0} \pi^{0}\right\rangle & =\sqrt{\frac{1}{3}}|0\rangle-\sqrt{\frac{2}{3}}|2\rangle,
\end{aligned}
$$

where $|I\rangle$ is the state of the two pions with the angular momentum equal to zero and the total isotopic spin equal to $I$.

The presentation of the states of two pions as a superposition of states with definite total isotopic spin will allow us to take into account the approximate $|\Delta I|=\frac{1}{2}$ rule, which is valid for the nonleptonic decays of the strange particles. For example, according to this rule the ratio of the total widths of the decays $K_{S} \rightarrow \pi^{+} \pi^{-}$and $K_{S} \rightarrow \pi^{0} \pi^{0}$ must be equal to 2 . We see from the Table I that this prediction is satisfied with the accuracy of about $10 \%$.

Let us consider now the parameters $\eta_{+-}$and $\eta_{00}$. From (176) and (179) we have

$$
\eta_{+-}=\frac{\left\langle 0|T| K_{L}^{0}\right\rangle+\frac{1}{\sqrt{2}}\left\langle 2|T| K_{L}^{0}\right\rangle}{\left\langle 0|T| K_{S}^{0}\right\rangle+\frac{1}{\sqrt{2}}\left\langle 2|T| K_{S}^{0}\right\rangle} .
$$

The amplitude $\left\langle 0|T| K_{S}^{0}\right\rangle$ is $C P$-allowed and is allowed by the $\left.|\Delta I|=\frac{1}{2}\right)$ rule. If we divide numerator and denominator of (180) by this "large" amplitude in the linear over small parameters approximation we find

$$
\eta_{+-} \simeq \epsilon+\epsilon^{\prime},
$$


where

$$
\epsilon=\frac{\left\langle 0|T| K_{L}^{0}\right\rangle}{\left\langle 0|T| K_{S}^{0}\right\rangle}
$$

and

$$
\epsilon^{\prime}=\frac{1}{\sqrt{2}}\left(\frac{\left\langle 2|T| K_{L}^{0}\right\rangle}{\left\langle 0|T| K_{S}^{0}\right\rangle}-\epsilon \frac{\left\langle 2|T| K_{S}^{0}\right\rangle}{\left\langle 0|T| K_{S}\right\rangle}\right) .
$$

Analogously, for $\eta_{00}$ we obtain

$$
\eta_{00} \simeq \epsilon-2 \epsilon^{\prime}
$$

Thus, we can characterize the $C P$ violation in the decays (169) by the parameters $\epsilon$ and $\epsilon^{\prime}$. 13 From (183), we can expect that $\left|\epsilon^{\prime}\right| \ll|\epsilon|$. As we will see later, experimental data confirm this expectation.

All existing data on the investigation of effects of the $C P$ violation in decays of $K_{L}^{0}$ are described by the Standard Model with three families of quarks. It is interesting, however, to mention other alternatives. Historically it was important the hypothesis of a superweak interaction [7]. It was suggested in [7] that effects of the $C P$ violation in the decays (169) can be explained by existence of a new interaction which violates $C P$ and changes the strangeness by two units.

In order to explain the idea of the superweak model, let us consider the relation (171). Taking into account (154) and (156) we find

$$
\bar{\epsilon}=\frac{\mathcal{H}_{12}-\mathcal{H}_{21}}{\left(\sqrt{\mathcal{H}_{12}}+\sqrt{\mathcal{H}_{21}}\right)^{2}}
$$

Obviously we have

$$
\left(\sqrt{\mathcal{H}_{12}}+\sqrt{\mathcal{H}_{21}}\right)^{2}=\bar{\epsilon}^{2}\left(\sqrt{\mathcal{H}_{12}}+\sqrt{\mathcal{H}_{21}}\right)^{2}+4 \sqrt{\mathcal{H}_{12} \mathcal{H}_{21}} .
$$

From (153) and (186) we find

$$
\left(\sqrt{\mathcal{H}_{12}}+\sqrt{\mathcal{H}_{21}}\right)^{2}=-\frac{2\left(\lambda_{L}-\lambda_{S}\right)}{1-\bar{\epsilon}^{2}}
$$

Thus, we have

$$
\frac{\bar{\epsilon}}{1-\bar{\epsilon}^{2}}=-\frac{\mathcal{H}_{12}-\mathcal{H}_{21}}{2\left(\lambda_{L}-\lambda_{S}\right)}
$$

\footnotetext{
${ }^{13}$ Let us notice that parameters $\epsilon$ and $\epsilon^{\prime}$ do not depend on arbitrary phases of $\left|K^{0}\right\rangle$ and $\left|\bar{K}^{0}\right\rangle$.
} 
Taking into account that $|\bar{\epsilon}| \sim 2 \cdot 10^{-3}$ and $\frac{\Gamma_{L}}{\Gamma_{S}} \ll 1$, from (188) we find the following relation

$$
\bar{\epsilon} \simeq-\frac{\mathcal{H}_{12}-\mathcal{H}_{21}}{2\left(\Delta m+\frac{i}{2} \Gamma_{S}\right)},
$$

where $\Delta m=m_{L}-m_{S}$.

It is shown in the Appendix B that in the effective Hamiltonian $\mathcal{H}$ enters linear in interaction term (see (B-32) $)$. Taking into account that the usual weak interaction Hamiltonian changes the strangeness by one unit, we have

$$
\left\langle K^{0}\left|H_{W}\right| \bar{K}^{0}\right\rangle=\left\langle K^{0}\left|H_{S W}\right| \bar{K}^{0}\right\rangle \neq\left\langle\bar{K}^{0}\left|H_{S W}\right| K^{0}\right\rangle,
$$

where $H_{S W}$ is a Hamiltonian which violates $C P$ and changes the strangeness by two units. It is evident from (189) that if such interaction exists the parameter $\bar{\epsilon}$ is different from zero.

In order to estimate the effective constant $G_{S W}$, which characterize the interaction $H_{S W}$, we will use the relation (189). Taking into account that $\Delta m \simeq \frac{1}{2} \Gamma_{S}$ we have

$$
|\bar{\epsilon}| \simeq \frac{G_{S W}}{G_{F}^{2} m_{K}^{2}} \approx 10^{-3},
$$

where $G_{F} \simeq 10^{-5} \frac{1}{m_{p}^{2}}$ is the Fermi constant and $m_{K}$ is the mass of the kaon. Thus, effects of the $C P$ violation in the decays of $K_{L}$ can be explained if the constant of $|\Delta S|=2$ interaction which violate $C P$ is given by $G_{S W} \approx$ $10^{-9} G_{F}$, i.e. is much smaller than the Fermi constant. This is the reason why this interaction is called superweak.

Let us consider the parameters $\eta_{+-}$and $\eta_{00}$ in the case of the superweak interaction. From (175) and (176) we have

$$
\eta_{+-}=\frac{\left\langle\pi^{+} \pi^{-}|T| K_{2}\right\rangle+\bar{\epsilon}\left\langle\pi^{+} \pi^{-}|T| K_{1}\right\rangle}{\left\langle\pi^{+} \pi^{-}|T| K_{1}\right\rangle+\bar{\epsilon}\left\langle\pi^{+} \pi^{-}|T| K_{2}\right\rangle} .
$$

In the superweak model $\left\langle\pi^{+} \pi^{-}|T| K_{2}\right\rangle \simeq 0$. We have

$$
\eta_{+-}=\bar{\epsilon}
$$

Analogously, for the decay $K_{L} \rightarrow \pi^{0} \pi^{0}$ we find

$$
\eta_{00}=\bar{\epsilon} .
$$


Thus, if the superweak interaction is the origin of the effects of the $C P$ violation, observed in the decays $K_{L} \rightarrow \pi \pi$, we would have

$$
\eta_{+-}=\eta_{00}
$$

From (181), (184) and (195) we conclude that in case of the superweak interaction $\epsilon^{\prime}=0$.

Taking into account linear in $\frac{\epsilon^{\prime}}{\epsilon}$ terms, from (181) and (184) we have in the general case

$$
\left|\eta_{+-}\right|^{2} \simeq|\epsilon|^{2}\left(1+2 \operatorname{Re} \frac{\epsilon^{\prime}}{\epsilon}\right),\left|\eta_{00}\right|^{2} \simeq|\epsilon|^{2}\left(1-4 \operatorname{Re} \frac{\epsilon^{\prime}}{\epsilon}\right) .
$$

From this relations we find

$$
\operatorname{Re} \frac{\epsilon^{\prime}}{\epsilon}=\frac{1}{6}\left(1-\frac{\left|\eta_{00}\right|^{2}}{\left|\eta_{+-}\right|^{2}}\right) .
$$

The ratio $\frac{\left|\eta_{0}\right|^{2}}{\left|\eta_{-}\right|^{2}}$ was measured in spectacular NA48 [8] and KTeV [9] experiments. It was found from the data of these experiments that

$$
\operatorname{Re} \frac{\epsilon^{\prime}}{\epsilon}=(14.7 \pm 2.2) 10^{-4}(\mathrm{NA} 48), \quad \operatorname{Re} \frac{\epsilon^{\prime}}{\epsilon}=(20.7 \pm 2.8) 10^{-4}(\mathrm{KTeV}) .
$$

Thus, it was proved that the parameter $\epsilon^{\prime}$ is different from zero and is much smaller than the parameter $\epsilon$. Therefore, it was proved that effects of the $C P$ violation, observed in the decays (169), can not be explained by the superweak interaction. It was shown that the measured value of the parameter Re $\frac{\epsilon^{\prime}}{\epsilon}$ can be explained by the SM (see[26]).

We will consider now the expressions (182) and (183) for $\epsilon$ and $\epsilon^{\prime}$. Neglecting quadratic in small parameters terms, for $\epsilon$ we obtain the following expression

$$
\epsilon \simeq \bar{\epsilon}+\frac{\left\langle 0|T| K_{2}\right\rangle}{\left\langle 0|T| K_{1}\right\rangle}
$$

For the parameter $\epsilon^{\prime}$ we find

$$
\begin{aligned}
\epsilon^{\prime} & =\frac{1}{\sqrt{2}}\left[\frac{\left\langle 2|T| K_{2}\right\rangle}{\left\langle 0|T| K_{1}\right\rangle}+(\bar{\epsilon}-\epsilon) \frac{\left\langle 2|T| K_{1}\right\rangle}{\left\langle 0|T| K_{1}\right\rangle}\right] \\
& =\frac{1}{\sqrt{2}} \frac{\left\langle 2|T| K_{1}\right\rangle}{\left\langle 0|T| K_{1}\right\rangle}\left[\frac{\left\langle 2|T| K_{2}\right\rangle}{\left\langle 2|T| K_{1}\right\rangle}-\frac{\left\langle 0|T| K_{2}\right\rangle}{\left\langle 0|T| K_{1}\right\rangle}\right],
\end{aligned}
$$


where

$$
\left\langle I|T| K_{1,2}\right\rangle=\frac{1}{\sqrt{2}}\left(\left\langle I|T| K^{0}\right\rangle \pm\left\langle I|T| \bar{K}^{0}\right\rangle\right), \quad I=0,2 .
$$

Let us consider the matrix elements $\left\langle I|T| K^{0}\right\rangle$ and $\left\langle I|T| \bar{K}^{0}\right\rangle$. From the unitarity of the S-matrix we have

$$
S^{\dagger} T=T^{\dagger}
$$

From this relation we find

$$
\left\langle I\left|S^{\dagger} T\right| K^{0}\right\rangle=\sum_{n}\left\langle I\left|S^{\dagger}\right| n\right\rangle\left\langle n|T| K^{0}\right\rangle=\left\langle K^{0}|T| I\right\rangle^{*} .
$$

In the sum over intermediate states $|n\rangle$ enter $|I\rangle,|\pi \pi \pi\rangle,|\pi \pi \gamma\rangle$ and other states. The main contribution gives the two-pion state $|I\rangle$ : the state of three pions is forbidden by the conservation of $G$-parity in the strong interaction, contributions of other states are suppressed by phase space factor and by $\alpha$. We have

$$
\left\langle I\left|S^{\dagger}\right| I\right\rangle=e^{-2 i \delta_{I}}
$$

where $\delta_{I}$ is the phase of the $\pi-\pi$-scattering in the state with the total isotopic spin equal to $I$, the angular momentum equal to zero and the energy in the center of mass system equal to $m_{K}$.

Further, from the CPT invariance it follows that

$$
\left\langle K^{0}|T| I\right\rangle=\left\langle I|T| \bar{K}^{0}\right\rangle .
$$

Thus, from the unitarity of the $S$-matrix and the $C P T$ invariance we find that the matrix elements $\left\langle I|T| K^{0}\right\rangle$ and $\left\langle I|T| \bar{K}^{0}\right\rangle$ are connected by the following relation

$$
e^{-2 i \delta_{I}}\left\langle I|T| K^{0}\right\rangle=\left\langle I|T| \bar{K}^{0}\right\rangle^{*} .
$$

Let us introduce the complex amplitudes $A_{I}$ and $\bar{A}_{I}$ in the following way

$$
\left\langle I|T| K^{0}\right\rangle=e^{i \delta_{I}} A_{I}, \quad\left\langle I|T| \bar{K}^{0}\right\rangle=e^{i \delta_{I}} \bar{A}_{I}
$$

From the relation (206) we find that

$$
\bar{A}_{I}=A_{I}^{*}
$$

Thus, we have

$$
\left\langle I|T| K^{0}\right\rangle=e^{i \delta_{I}} A_{I}, \quad\left\langle I|T| \bar{K}^{0}\right\rangle=e^{i \delta_{I}} A_{I}^{*} .
$$


In the case of the $C P$ conservation we have that

$$
\left\langle I|T| K^{0}\right\rangle=\left\langle I|T| \bar{K}^{0}\right\rangle
$$

and

$$
A_{I}=A_{I}^{*} .
$$

Let us return now back to relations (199) and (200). Taking into account (209) we find

$$
\epsilon=\bar{\epsilon}+i \frac{\operatorname{ImA}_{0}}{\operatorname{ReA}_{0}} .
$$

and

$$
\epsilon^{\prime}=\frac{1}{\sqrt{2}} e^{i\left(\delta_{2}-\delta_{0}+\frac{\pi}{2}\right)} \frac{\operatorname{ReA}_{2}}{\operatorname{ReA}_{0}}\left[\frac{\operatorname{ImA} A_{2}}{\operatorname{ReA}_{2}}-\frac{\operatorname{ImA}_{0}}{\operatorname{ReA}_{0}}\right]
$$

From these relations we can conclude the following:

1.

$$
\phi_{\epsilon^{\prime}}=\delta_{2}-\delta_{0}+\frac{\pi}{2},
$$

where $\phi_{\epsilon^{\prime}}=\arg \epsilon^{\prime}$ is the phase of the parameter $\epsilon^{\prime}$. From analysis of the $\pi-\pi$ scattering data it was obtained [65]

$$
\delta_{2}-\delta_{0}+\frac{\pi}{2}=(42.3 \pm 1.5)^{\circ}
$$

2.

$$
\operatorname{Re} \epsilon=\operatorname{Re} \bar{\epsilon}
$$

The parameter $\bar{\epsilon}$ depends on the choice of arbitrary phase of the $\left|K^{0}\right\rangle$ and $\left|\bar{K}^{0}\right\rangle$ states. We see from (216) that $\operatorname{Re} \bar{\epsilon}$ is rephrase invariant quantity.

For the phase of the parameter $\epsilon$ the following relation holds

$$
\phi_{\epsilon} \simeq \arctan \frac{2 \Delta m}{\Gamma_{S}},
$$

where $\phi_{\epsilon}=\arg \epsilon$. This relation is based on the Bell-Steinberger unitarity relation which we derive now. We have

$$
\mathcal{H} a_{L}=\lambda_{L} a_{L}, \quad a_{S}^{\dagger} \mathcal{H}^{\dagger}=\lambda_{S}^{*} a_{S}^{\dagger} .
$$


If we multiply the first equation by $a_{S}^{\dagger}$ from the left and the second one by $a_{L}$ from the right and subtract from the first relation the second one we find

$$
\left(a_{S}^{\dagger} \Gamma a_{L}\right)=i\left(\lambda_{L}-\lambda_{S}^{*}\right)\left(a_{S}^{\dagger} a_{L}\right)
$$

This relation can be rewritten in the form

$$
\left\langle K_{S}|\Gamma| K_{L}\right\rangle=i\left(\lambda_{L}-\lambda_{S}^{*}\right)\left\langle K_{S} \mid K_{L}\right\rangle
$$

The relation (220) is the Bell-Steinberger unitarity relation. For the left-hand side of this equation we have

$$
\left\langle K_{S}|\Gamma| K_{L}\right\rangle=2 \pi \sum_{i}\left\langle K_{S}\left|H_{W}\right| i\right\rangle\left\langle i\left|H_{W}\right| K_{L}\right\rangle \delta\left(E_{i}-m\right) .
$$

In the sum over intermediate states $|i\rangle$ main contribution give two-pion states. Taking into account these states we have

$$
\left\langle K_{S}|\Gamma| K_{L}\right\rangle \simeq \eta_{+-} \Gamma\left(K_{S} \rightarrow \pi^{+} \pi^{-}\right)+\eta_{00} \Gamma\left(K_{S} \rightarrow \pi^{0} \pi^{0}\right) .
$$

Now, according to $|\Delta I|=1 / 2$ rule we have

$$
\Gamma\left(K_{S} \rightarrow \pi^{+} \pi^{-}\right) \simeq 2 \Gamma\left(K_{S} \rightarrow \pi^{0} \pi^{0}\right) .
$$

From (181), (184), (222) and (223) we find

$$
\left\langle K_{S}|\Gamma| K_{L}\right\rangle \simeq\left(\frac{2}{3} \eta_{+-}+\frac{1}{3} \eta_{00}\right) \Gamma_{S}=\epsilon \Gamma_{S},
$$

where $\Gamma_{S}$ is the total width of $K_{S}^{0}$-meson. Further, we find

$$
\left\langle K_{S} \mid K_{L}\right\rangle=\frac{2 \operatorname{Re} \bar{\epsilon}}{1+|\bar{\epsilon}|^{2}} \simeq 2 \operatorname{Re} \bar{\epsilon}=2 \operatorname{Re} \epsilon
$$

From (220), (224) and (225) we obtain the following relation

$$
\epsilon \Gamma_{S}=2\left(i \Delta m+\frac{1}{2} \Gamma_{S}\right) \operatorname{Re} \epsilon
$$

If we take the real part of (226) we obtain identity. From the imaginary part of (226) we find the following relation

$$
\frac{\operatorname{Im} \epsilon}{\operatorname{Re} \epsilon}=\tan \phi_{\epsilon}=\frac{2 \Delta m}{\Gamma_{S}} .
$$


Thus, the phase of the parameter $\epsilon$ is given by the relation (217).

The mass difference $\Delta m$ and the width $\Gamma_{S}$ are connected by the empirical relation $\Delta m \simeq \frac{1}{2} \Gamma_{S}$. Thus, $\phi_{\epsilon} \simeq \pi / 4$. The experimental data are in an agreement with this prediction of the theory. We have [32]

$$
\phi_{\epsilon}=(43.5 \pm 0.7)^{\circ} .
$$

From (215) and (228) it follows that phases of the parameters $\epsilon$ and $\epsilon^{\prime}$ are approximately equal

$$
\phi_{\epsilon} \simeq \phi_{\epsilon^{\prime}} .
$$

Up to now we considered effects of the $C P$ violation in the two-pion decays of $K_{L}^{0}$-meson. Effects of the $C P$ violation were observed also in the semi-leptonic decays

$$
K_{L}^{0} \rightarrow \pi^{-} l^{+} \nu_{l}, \quad K_{L}^{0} \rightarrow \pi^{+} l^{-} \bar{\nu}_{l} .
$$

Let us determine the $C P$ asymmetry

$$
A_{L}=\frac{\Gamma\left(K_{L}^{0} \rightarrow \pi^{-} l^{+} \nu_{l}\right)-\Gamma\left(K_{L}^{0} \rightarrow \pi^{+} l^{-} \bar{\nu}_{l}\right)}{\Gamma\left(K_{L}^{0} \rightarrow \pi^{-} l^{+} \nu_{l}\right)+\Gamma\left(K_{L}^{0} \rightarrow \pi^{+} l^{-} \bar{\nu}_{l}\right)},
$$

where $\Gamma\left(K_{L} \rightarrow \pi^{-} l^{+} \nu_{l}\right)$ and $\Gamma\left(K_{L}^{0} \rightarrow \pi^{+} l^{-} \bar{\nu}_{l}\right)$ are the total widths of the decays $K_{L}^{0} \rightarrow \pi^{-} l^{+} \nu_{l}$ and $K_{L} \rightarrow \pi^{+} l^{-} \bar{\nu}_{l}$. If the $C P$ is conserved the asymmetry $A_{L}$ is equal to zero. In fact, in this case initial state is the eigenstate of the operator of the $C P$ conjugation and final states are the $C P$ conjugated states. The probabilities of the transitions to such states must be equal in the case of the $C P$ conservation.

Let us consider the asymmetry $A_{L}$. The semi-leptonic decay of the $K^{0}$ meson, which is the bound state of $\bar{s}$ and $d$ quarks, is due to the transition $\bar{s} \rightarrow \bar{u}+l^{+}+\nu_{l}$. Analogously the decay of the $\bar{K}^{0}$-meson, which is the bound state of the $s$ and $\bar{d}$ quarks, is due the transition $s \rightarrow u+l^{-}+\bar{\nu}_{l}$. Thus, the decay $K^{0} \rightarrow \pi^{-} l^{+} \nu_{l}$ is allowed and decay $\bar{K}^{0} \rightarrow \pi^{-} l^{+} \nu_{l}$ is forbidden and the decay $\bar{K}^{0} \rightarrow \pi^{+} l^{-} \bar{\nu}_{l}$ is allowed and $K^{0} \rightarrow \pi^{+} l^{-} \bar{\nu}_{l}$ is forbidden. This corresponds to the $\Delta Q=\Delta S$ rule. Further, from the CPT invariance it follows that

$$
\left\langle\pi^{+} l^{-} \bar{\nu}_{l}|T| \bar{K}^{0}\right\rangle=\left\langle K^{0}|T| \pi^{-} l^{+} \nu_{l}\right\rangle \simeq\left\langle\pi^{-} l^{+} \nu_{l}|T| K^{0}\right\rangle^{*},
$$

where we took into account that in the Born approximation $T=T^{\dagger}$. 
From (172) and (232) we find

$$
A_{L}=\frac{|1+\bar{\epsilon}|^{2}-|1-\bar{\epsilon}|^{2}}{|1+\bar{\epsilon}|^{2}+|1-\bar{\epsilon}|^{2}}=\frac{2 \operatorname{Re} \bar{\epsilon}}{1+|\bar{\epsilon}|^{2}} \simeq 2 \operatorname{Re} \bar{\epsilon} .
$$

Now, taking into account (216), we finally have

$$
A_{L} \simeq 2 \operatorname{Re} \epsilon=2|\epsilon| \cos \phi_{\epsilon}
$$

From experimental data for the asymmetry $A_{L}$ it was found the value [32]

$$
A_{L}=(3.32 \pm 0.06) \cdot 10^{-3} \text {. }
$$

From (215) and (235) for the parameter $|\epsilon|$ was found the value

$$
|\epsilon|=(2.232 \pm 0.007) \cdot 10^{-3}
$$

In order to connect $|\epsilon|$ with parameters, characterizing $C K M$ mixing matrix, it is necessary to calculate quark box diagrams which determine the amplitude of $K^{0} \rightarrow \bar{K}^{0}$ transition. Taking into account the $Q C D$ corrections for the parameter $|\epsilon|$ it was found the following expression

$$
|\epsilon|=A^{2} a \bar{\eta}\left[A^{2} b(1-\bar{\rho})+c\right]
$$

where $a, b$ and $c$ are given in [26]. The equation (237) gives hyperbola in $\bar{\rho}, \bar{\eta}$ plane. It is used in the standard unitarity triangle fit which we will discuss later.

\section{$9 C P$ violation and mixing in $B^{0}-\bar{B}^{0}$ system}

We will consider in this section effects of the $C P$ violation in decays of the mixed $B^{0}-\bar{B}^{0}$ system. These effects were investigated in details in the BaBar and the Belle experiments at the asymmetric B-factories and in the D0 and the CDF experiments at the Fermilab. At the B-factories $B_{d}^{0}$ and $\bar{B}_{d}^{0}$ mesons are resonansly produced in decays of $\Upsilon(4 S)$.

The states of $B_{H}^{0}$ and $B_{L}^{0}$ mesons, particles with definite masses and widths, are given by the following relations

$$
\left|B_{H, L}^{0}\right\rangle=p\left|B^{0}\right\rangle \mp q\left|\bar{B}^{0}\right\rangle,
$$


where $p$ and $q$ are connected with non diagonal elements of the effective Hamiltonian by the relations (154) and (155). Let us stress that we have choosen arbitrary phases of the states $\left|B^{0}\right\rangle$ and $\left|\bar{B}^{0}\right\rangle$ in such a way that $\left|\bar{B}^{0}\right\rangle=C P\left|B^{0}\right\rangle$.

The states $\left|B_{H, L}^{0}\right\rangle$ are eigenstates of the effective Hamiltonian $\mathcal{H}$ with eigenvalues

$$
\mu_{H, L}=m_{H, L}-i \frac{1}{2} \Gamma_{H, L}
$$

Here $m_{H, L}$ and $\Gamma_{H, L}$ are masses and total decay widths of $B_{H, L}^{0}$ mesons. Because of the large difference in the lifetimes of the short-lived and longlived kaons it is possible to produce beams of $K_{L}^{0}$-mesons. In the case of the $B^{0}$ mesons the situation is different. The lifetimes of $B_{H}^{0}$ and $B_{L}^{0}$ are quite close. Only mixtures of $B_{H}^{0}$ and $B_{L}^{0}$ can be studied in experiments.

Let us obtain first the mixed states which are the result of the evolution of the initial (at $t=0)\left|B^{0}\right\rangle$ and $\left|\bar{B}^{0}\right\rangle$ states. From (238) we have

$$
\left|B^{0}\right\rangle=\frac{1}{2 p}\left(\left|B_{H}^{0}\right\rangle+\left|B_{L}^{0}\right\rangle\right), \quad\left|\bar{B}^{0}\right\rangle=\frac{1}{2 q}\left(-\left|B_{H}^{0}\right\rangle+\left|B_{L}^{0}\right\rangle\right) .
$$

From (240) we find

$$
\left|B^{0}(t)\right\rangle=\frac{1}{2 p}\left(e^{-i \mu_{H} t}\left|B_{H}^{0}\right\rangle+e^{-i \mu_{L} t}\left|B_{L}^{0}\right\rangle\right)=g_{+}(t)\left|B^{0}\right\rangle-\frac{q}{p} g_{-}(t)\left|\bar{B}^{0}\right\rangle
$$

and

$$
\left|\bar{B}^{0}(t)\right\rangle=\frac{1}{2 q}\left(-e^{-i \mu_{H} t}\left|B_{H}^{0}\right\rangle+e^{-i \mu_{L} t}\left|B_{L}^{0}\right\rangle\right)=-\frac{p}{q} g_{-}(t)\left|B^{0}\right\rangle+g_{+}(t)\left|\bar{B}^{0}\right\rangle .
$$

Here

$$
g_{ \pm}(t)=\frac{1}{2}\left(e^{-i \mu_{H} t} \pm e^{-i \mu_{L} t}\right) .
$$

Let us present $\mu_{H, L}$ in the form

$$
\mu_{H}=\mu+\frac{1}{2} \Delta \mu, \quad \mu_{L}=\mu-\frac{1}{2} \Delta \mu .
$$

Here

$$
\mu=\frac{\mu_{H}+\mu_{L}}{2}=m-i \frac{1}{2} \Gamma, \Delta \mu=\mu_{H}-\mu_{L}=\Delta m-i \frac{1}{2} \Delta \Gamma,
$$


where

$$
m=\frac{m_{H}+m_{L}}{2}, \Gamma=\frac{\Gamma_{H}+\Gamma_{L}}{2}, \Delta m=m_{H}-m_{L}, \Delta \Gamma=\Gamma_{H}-\Gamma_{L} .
$$

Notice that by the definition $\Delta m>0$. From (243) and (244) for the functions $g_{ \pm}(t)$ we obtain the following expressions

$$
g_{ \pm}(t)=\frac{1}{2} e^{-i \mu t}\left(e^{-i \frac{1}{2} \Delta \mu t} \pm e^{i \frac{1}{2} \Delta \mu t}\right) .
$$

Let us consider the decays of $B^{0}$ and $\bar{B}^{0}$ into a state $|f\rangle$ which is the eigenstate of the operator of the $C P$ conjugation

$$
C P|f\rangle= \pm|f\rangle \text {. }
$$

From (241) for the transition amplitude we find

$$
\left\langle f|T| B^{0}(t)\right\rangle=\left\langle f|T| B^{0}\right\rangle\left(g_{+}(t)-\lambda_{f} g_{-}(t)\right)
$$

where

$$
\lambda_{f}=\frac{q}{p} \frac{\left\langle f|T| \bar{B}^{0}\right\rangle}{\left\langle f|T| B^{0}\right\rangle} .
$$

For the transition amplitude $\left\langle f|T| \bar{B}^{0}(t)\right\rangle$ we have

$$
\left\langle f|T| \bar{B}^{0}(t)\right\rangle=\frac{p}{q}\left\langle f|T| B^{0}\right\rangle\left(-g_{-}(t)+\lambda_{f} g_{+}(t)\right) .
$$

From (249) we find that the transition probability is given by the expression

$$
\Gamma\left(B^{0}(t) \rightarrow f\right)=\Gamma\left(B^{0} \rightarrow f\right)\left(\left|g_{+}(t)\right|^{2}+\left|\lambda_{f}\right|^{2}\left|g_{-}(t)\right|^{2}-2 \operatorname{Re} \lambda_{\mathrm{f}} \mathrm{g}_{-}(\mathrm{t}) \mathrm{g}_{+}^{*}(\mathrm{t})\right) \text {. }
$$

Further, from (247) we have

$$
\left|g_{ \pm}(t)\right|^{2}=\frac{1}{2} e^{-\Gamma t}\left(\cosh \frac{1}{2} \Delta \Gamma t \pm \cos \Delta m t\right)
$$

and

$$
g_{-}(t) g_{+}^{*}(t)=-\frac{1}{2} e^{-\Gamma t}\left(\sinh \frac{1}{2} \Delta \Gamma t+i \sin \Delta m t\right) .
$$

From (252), (253) and (254) for the transition probability $\Gamma\left(B^{0}(t) \rightarrow f\right)$ we obtain the following expression

$$
\begin{aligned}
\Gamma\left(B^{0}(t) \rightarrow f\right) & =\frac{1}{2} e^{-\Gamma t} \Gamma\left(B^{0} \rightarrow f\right)\left(1+\left|\lambda_{f}\right|^{2}\right)\left(\cosh \frac{1}{2} \Delta \Gamma t\right. \\
& \left.+C_{f} \cos \Delta m t+D_{f} \sinh \frac{1}{2} \Delta \Gamma t-S_{f} \sin \Delta m t\right),
\end{aligned}
$$


where

$$
C_{f}=\frac{1-\left|\lambda_{f}\right|^{2}}{\left(1+\left|\lambda_{f}\right|^{2}\right)}, \quad D_{f}=\frac{2 \operatorname{Re} \lambda_{\mathrm{f}}}{\left(1+\left|\lambda_{f}\right|^{2}\right)}, S_{f}=\frac{2 \operatorname{Im} \lambda_{\mathrm{f}}}{\left(1+\left|\lambda_{f}\right|^{2}\right)}
$$

It is obvious from (256) that parameters $C_{f}, D_{f}$ and $S_{f}$ satisfy the relation

$$
C_{f}^{2}+D_{f}^{2}+S_{f}^{2}=1
$$

For the transition probability $\Gamma\left(\bar{B}^{0}(t) \rightarrow f\right)$ from (251) we find

$\Gamma\left(\bar{B}^{0}(t) \rightarrow f\right)=\left|\frac{p}{q}\right|^{2} \Gamma\left(B^{0} \rightarrow f\right)\left(\left|g_{-}(t)\right|^{2}+\left|\lambda_{f}\right|^{2}\left|g_{+}(t)\right|^{2}-2 \operatorname{Re} \lambda_{\mathrm{f}} \mathrm{g}_{+}(\mathrm{t}) \mathrm{g}_{-}^{*}(\mathrm{t})\right)$

From (253), (254) and (258) for the probability $\Gamma\left(\bar{B}^{0}(t) \rightarrow f\right)$ we obtain the following expression

$$
\begin{aligned}
\Gamma\left(\bar{B}^{0}(t) \rightarrow f\right) & =\frac{1}{2} e^{-\Gamma t}\left|\frac{p}{q}\right|^{2} \Gamma\left(B^{0} \rightarrow f\right)\left(1+\left|\lambda_{f}\right|^{2}\right)\left(\cosh \frac{1}{2} \Delta \Gamma t\right. \\
& \left.-C_{f} \cos \Delta m t+D_{f} \sinh \frac{1}{2} \Delta \Gamma t+S_{f} \sin \Delta m t\right) .
\end{aligned}
$$

If $C P$ is conserved $\left\langle f|T| B^{0}\right\rangle= \pm\left\langle f|T| \bar{B}^{0}\right\rangle, p=q$ and $\lambda_{f}= \pm 1$. In this case we have: $\Gamma\left(B^{0}(t) \rightarrow f\right)=\Gamma\left(\bar{B}^{0}(t) \rightarrow f\right)=e^{-\Gamma_{H} t}\left(e^{-\Gamma_{L} t}\right) \Gamma\left(B^{0} \rightarrow f\right)$.

The quantity $\lambda_{f}$, which determine the time dependence of the probabilities $\Gamma\left(B^{0}(t) \rightarrow f\right)$ and $\Gamma\left(\bar{B}^{0}(t) \rightarrow f\right)$, does not depend on arbitrary phases of the states of $B^{0}, \bar{B}^{0}$ and $f$. In fact, let us consider the states

$$
\left|B^{0}\right\rangle^{\prime}=e^{i \alpha}\left|B^{0}\right\rangle,\left|\bar{B}^{0}\right\rangle^{\prime}=e^{-i \alpha}\left|\bar{B}^{0}\right\rangle,|f\rangle^{\prime}=e^{i \beta}|f\rangle
$$

where $\alpha$ and $\beta$ are arbitrary constants. From (154) and (156) we have

$$
q^{\prime}=e^{i \alpha} q, \quad p^{\prime}=e^{-i \alpha} p .
$$

From (250), (260) and (261) we find

$$
\lambda_{f}^{\prime}=\frac{q^{\prime}}{p^{\prime}} \frac{\left\langle f|T| \bar{B}^{0}\right\rangle^{\prime}}{\prime\left\langle f|T| B^{0}\right\rangle^{\prime}}=\lambda_{f} .
$$

Let us consider now the matrix element $\Gamma_{12}$. From (B-35) we have

$$
\Gamma_{12}=2 \pi \sum_{i}\left\langle B^{0}\left|H_{W}\right| i\right\rangle\left\langle i\left|H_{W}\right| \bar{B}^{0}\right\rangle \delta\left(E-m_{B}\right)
$$


From this expression follows that the contribution to $\Gamma_{12}$ give intermediate states $|i\rangle$ in which both $B^{0}$ and $\bar{B}^{0}$ mesons can decay. In the Standard Model transitions to such states are strongly suppressed (see, for example, [26]). Thus, in the SM we have

$$
\left|\Gamma_{12}\right| \ll\left|M_{12}\right| .
$$

From (153) we find

$$
\Delta \mu=\Delta m-i \frac{1}{2} \Delta \Gamma=2\left|M_{12}\right| \sqrt{\left(1-\frac{i}{2} \frac{\Gamma_{12}}{M_{12}}\right)\left(1-\frac{i}{2} \frac{\Gamma_{12}^{*}}{M_{12}^{*}}\right)}
$$

Taking into account (264), from this expression we obtain

$$
\Delta m-i \frac{1}{2} \Delta \Gamma=2\left|M_{12}\right|\left(1-\frac{i}{2} \operatorname{Re} \frac{\Gamma_{12}}{\mathrm{M}_{12}}\right)+\mathrm{O}\left(\left|\frac{\Gamma_{12}}{\mathrm{M}_{12}}\right|^{2}\right)
$$

Thus, we have

$$
\Delta m \simeq 2\left|M_{12}\right|, \quad \Delta \Gamma \simeq 2 \operatorname{Re} \frac{\Gamma_{12}}{\mathrm{M}_{12}}\left|\mathrm{M}_{12}\right|
$$

Let us consider now the mixing parameter $\frac{q}{p}$. We have

$$
\frac{q}{p}=\sqrt{\frac{H_{21}}{H_{12}}}=-\frac{2 H_{21}}{\Delta \mu} .
$$

Neglecting terms of the order $O\left(\left|\frac{\Gamma_{12}}{M_{12}}\right|^{2}\right)$, from (266) and (268) we find

$$
\frac{q}{p} \simeq-\frac{M_{12}^{*}\left(1-\frac{i}{2} \frac{\Gamma_{12}^{*}}{M_{12}^{*}}\right)}{\left|M_{12}\right|\left(1-\frac{i}{2} \operatorname{Re} \frac{\Gamma_{12}}{\mathrm{M}_{12}}\right)}=\simeq-\frac{M_{12}^{*}}{\left|M_{12}\right|}\left(1-\frac{1}{2} \operatorname{Im} \frac{\Gamma_{12}}{\mathrm{M}_{12}}\right) \text {. }
$$

Let us determine the $C P$ asymmetry in the case of the decays of $B^{0}$ and $\bar{B}^{0}$ into the state $f$ which is the eigenstate of the operator of the $C P$ conjugation

$$
A_{f}^{C P}(t)=\frac{\Gamma\left(\bar{B}^{0}(t) \rightarrow f\right)-\Gamma\left(B^{0}(t) \rightarrow f\right)}{\Gamma\left(\bar{B}^{0}(t) \rightarrow f\right)+\Gamma\left(B^{0}(t) \rightarrow f\right)} .
$$

In the SM in the case of the $B_{d}$-mesons

$$
\frac{\Delta \Gamma_{d}}{\Gamma_{d}} \ll 1 .
$$


For example, in [66] it was found

$$
\frac{\Delta \Gamma_{d}}{\Gamma_{d}}=\left(40.9_{-9.9}^{+8.9}\right) \cdot 10^{-4} \cdot \frac{\Delta \Gamma_{s}}{\Gamma_{s}}=0.127 \pm 0.024
$$

We will consider $B_{d}^{0}-\bar{B}_{d}^{0}$-system. We can neglect in (255) and (259) $\Delta \Gamma_{d}$. We can also neglect $\operatorname{Im} \frac{\Gamma_{12}}{\mathrm{M}_{12}}$ in (269). Thus, we have $\left|\frac{q}{p}\right| \simeq 1$ and from (255) and (259) for the asymmetry we find the expression

$$
A_{f}^{C P}(t)=-C_{f} \cos \Delta m t+S_{f} \sin \Delta m t .
$$

In conclusion we will consider the following decays

$$
B_{d}^{0}\left(\bar{B}_{d}^{0}\right) \rightarrow J / \Psi+K_{S, L}^{0}
$$

These decay modes are called golden by the reasons which will be clear later.

Final $J / \Psi$ and $K_{S, L}^{0}$ particles are in the state with $l=1$. Neglecting in the matrix elements of the decay small terms of the order of $\sim 10^{-3}$ we can put $\left|K_{S}\right\rangle \simeq\left|K_{1}\right\rangle$ and $\left|K_{L}\right\rangle \simeq\left|K_{2}\right\rangle$. Thus, we find

$$
C P\left|J / \Psi K_{S, L}^{0}\right\rangle=\eta_{S, L}\left|J / \Psi K_{S, L}^{0}\right\rangle,
$$

where $\eta_{S, L}=\mp 1$.

Matrix elements of the processes $\bar{B}_{d}^{0}\left(B_{d}^{0}\right) \rightarrow J / \Psi+K_{S . L}^{0}$ are determined by decays of the $b$-quark, which are governed by the tree and penguin electroweak diagrams. If we take into account $Q C D$ corrections, the matrix elements of the process $\bar{B}_{d}^{0} \rightarrow J / \Psi+K_{S, L}^{0}$ is given by the relation (see reviews [26, 25])

$$
\begin{aligned}
\left\langle J / \Psi K_{S, L}^{0}|T| \bar{B}_{d}^{0}\right\rangle & =\frac{G_{F}}{\sqrt{2}} \sum_{q=u, c} V_{q b} V_{q s}^{*}\left(\sum_{k=1,2} C_{k}(\mu)\left\langle J / \Psi K_{S, L}^{0}\left|O_{k}^{q s}\right| \bar{B}_{d}^{0}\right\rangle\right. \\
& \left.+\sum_{k=3}^{10} C_{k}(\mu)\left\langle J / \Psi K_{S, L}^{0}\left|O_{k}^{s}\right| \bar{B}_{d}^{0}\right\rangle\right)
\end{aligned}
$$

Here $C_{k}(\mu)$ are real Wilson coefficients, $O_{k}^{q s}$ are 4-quark current-current operators and $O_{k}^{s}$ are 4-quark penguin operators (for the definitions see, for example, [25]). 
For the matrix element of the process $B_{d}^{0} \rightarrow J / \Psi+K_{S, L}^{0}$ we have

$$
\begin{aligned}
\left\langle J / \Psi K_{S, L}^{0}|T| B_{d}^{0}\right\rangle & =\frac{G_{F}}{\sqrt{2}} \sum_{q=u, c} V_{q b}^{*} V_{q s}\left(\sum_{k=1,2} C_{k}(\mu)\left\langle J / \Psi K_{S, L}^{0}\left|\left(O_{k}^{q s}\right)^{\dagger}\right| B_{d}^{0}\right\rangle\right. \\
& \left.+\sum_{k=3}^{10} C_{k}(\mu)\left\langle J / \Psi K_{S, L}^{0}\left|\left(O_{k}^{s}\right)^{\dagger}\right| B_{d}^{0}\right\rangle\right) .
\end{aligned}
$$

Further, we have

$$
\left(O_{k}^{q s}\right)^{\dagger}=(C P)^{-1} O_{k}^{q s} C P, \quad\left(O_{k}^{s}\right)^{\dagger}=(C P)^{-1} O_{k}^{s} C P .
$$

From (275), (277) and (278) we find

$$
\begin{aligned}
\left\langle J / \Psi K_{S, L}^{0}|T| B_{d}^{0}\right\rangle & =\eta_{S, L} \frac{G_{F}}{\sqrt{2}} \sum_{q=u, c} V_{q b}^{*} V_{q s}\left(\sum_{k=1,2} C_{k}(\mu)\left\langle J / \Psi K_{S, L}^{0}\left|O_{k}^{q s}\right| \bar{B}_{d}^{0}\right\rangle\right. \\
& \left.+\sum_{k=3}^{10} C_{k}(\mu)\left\langle J / \Psi K_{S, L}^{0}\left|O_{k}^{s}\right| \bar{B}_{d}^{0}\right\rangle\right)
\end{aligned}
$$

Let us compare now the matrix elements $\left\langle J / \Psi K_{S, L}^{0}|T| \bar{B}_{d}^{0}\right\rangle$ and $\left\langle J / \Psi K_{S, L}^{0}|T| B_{d}^{0}\right\rangle$. The ratio of these matrix elements which enter into the expression for the parameter $\lambda_{J / \Psi K_{S, L}^{0}}$ (see (250) ) depends on the $C K M$ matrix elements and on the hadronic matrix elements. However, $\left|V_{u b} V_{u s}^{*}\right| \simeq 10^{-2}\left|V_{c b} V_{c s}^{*}\right|$. If we neglect in (276) and (279) the contribution of the terms proportional to $\left|V_{u b} V_{u s}^{*}\right|$ and take into account that the product $V_{c b}^{*} V_{c s}$ is real, we come to the following result

$$
\frac{\left\langle J / \Psi K_{S, L}^{0}|T| \bar{B}_{d}^{0}\right\rangle}{\left\langle J / \Psi K_{S, L}^{0}|T| B_{d}^{0}\right\rangle} \simeq \eta_{S, L} .
$$

For the parameter $\lambda_{J / \Psi K_{S, L}^{0}}$ we find

$$
\lambda_{J / \Psi K_{S, L}^{0}} \simeq \eta_{S, L} \frac{q}{p}
$$

Thus, in the case of the decays (274) the parameter $\lambda_{J / \Psi K_{S, L}^{0}}$ (practically) does not depend on hadronic uncertainties of the decay matrix elements.

The mixing parameter $\frac{q}{p}$ is given by the relation $\frac{q}{p} \simeq-\frac{M_{12}^{*}}{\mid M_{12}} \mid$ (see (187)). Main contribution to the box diagrams which determine matrix element $M_{12}$ gives the virtual $t$-quark. We have

$$
\frac{q}{p} \simeq-e^{\arg \left(V_{t b}^{*} V_{t d}\right)^{2}}
$$


From (133), (281) and (282) we find

$$
\lambda_{J / \Psi K_{S, L}^{0}} \simeq-\eta_{S, L} e^{2 i \beta}
$$

Thus, we have

$$
C_{J / \Psi K_{S, L}^{0}} \simeq 0, \quad S_{J / \Psi K_{S, L}^{0}} \simeq-\eta_{S, L} \sin 2 \beta
$$

From (273) and (284) for the asymmetry $A_{J / \Psi K_{S}^{0}}^{C P}(t)$ we find the following expression

$$
A_{J / \Psi K_{S}^{0}}^{C P}(t)=\sin 2 \beta \sin \Delta m t .
$$

Asymmetry $A_{J / \Psi K_{L}^{0}}^{C P}(t)$ differs by sign from $A_{J / \Psi K_{S}^{0}}^{C P}(t)$. We have

$$
A_{J / \Psi K_{L}^{0}}^{C P}(t)=-\sin 2 \beta \sin \Delta m t
$$

We came to an important conclusion: the measurement of $t$ dependence of the $C P$ asymmetries in the decays $\bar{B}_{d}^{0}\left(B_{d}^{0}\right) \rightarrow J / \Psi K_{S, L}^{0}$ allow to determine the angle $\beta$ in a model independent way [67].

The asymmetries $A_{C P}^{J / \Psi K_{L, S}^{0}}(t)$ were measured by the BaBar collaboration in experiments at the asymmetric B-factory at SLAC and by the Belle collaboration in experiments at the asymmetric B-factory at KEK. In these experiments the first evidence of the $C P$ violation in $B_{d}^{0}\left(\bar{B}_{d}^{0}\right)$ decays was found and the value of the parameter $\sin 2 \beta$ was determined [68, 69]. Recently the results of the measurement of the parameter $\sin 2 \beta$ in the experiments which were performed during 1999-2006 were published [70, 71].

At the asymmetric B-factories $B_{d}^{0}$-mesons are produced in the decay $\Upsilon(4 S) \rightarrow B_{d}^{0}+\bar{B}_{d}^{0}$. Flavor of a particle is determined by the tagging another particle. The proper time $t$ in (273) and other equations is given by difference between the proper time of reconstructed and tagged $B^{0}$ mesons: $t=t_{\mathrm{rec}}-t_{\mathrm{tag}}$. Because the $B_{d}^{0}$ and $\bar{B}_{d}^{0}$ mesons are practically at rest in $\Upsilon(4 S)$ rest frame we have $t_{\text {rec }}-t_{\mathrm{tag}}=\frac{z_{\mathrm{rec}}-z_{\mathrm{tag}}}{\beta \gamma c}$, where $z_{\text {rec }}$ and $z_{\mathrm{tag}}$ are positions of corresponding decay vertices and $\beta \gamma$ is the Lorenz boost of $\Upsilon(4 S)$.

In the BaBar experiment $(347.5 \pm 3.8) \cdot 10^{6} \Upsilon(4 S) \rightarrow B_{d}^{0}+\bar{B}_{d}^{0}$ decays were detected. For analysis were used decays determined by the transition $b \rightarrow c \bar{c} s$. The decays into the following egenstates of the $C P$ operator were analyzed: $J / \Psi K_{S}^{0}, J / \Psi K_{L}^{0}, \Psi(2 S) K_{S}^{0}, \chi_{c 1} K_{S}^{0}, \eta_{c} K_{S}^{0}$ and $J / \Psi K_{S}^{* 0}$. 
From analysis of the data the following result was obtained $[70] 14$

$$
\sin 2 \beta=0.710 \pm 0.034 \pm 0.019, \quad C=0.070 \pm 0.026 \pm 0.018
$$

In the Belle experiment $535 \cdot 10^{6} \Upsilon(4 S) \rightarrow B_{d}^{0}+\bar{B}_{d}^{0}$ decays were detected. From analysis of the decays into $J / \Psi K_{S}^{0}$ and $J / \Psi K_{L}^{0}$ states it was found [71].

$$
\sin 2 \beta=0.642 \pm 0.031 \pm 0.017, \quad C=-0.018 \pm 0.021 \pm 0.014 .
$$

Thus, the parameter $\sin 2 \beta$ is known today with accuracy about $5 \%$. This model independent result is very important for the unitarity triangle fit of the experimental data which we will discuss in the next section.

\section{Unitarity triangle test of the Standard Model}

Several groups [72, 73, 74, 75] analyze experimental data with the aim to perform the unitarity triangle test of the Standard Model and to search for effects of beyond the SM physics. Different groups use different statistical methods of the analysis of experimental data. We will present here some results of the UTfit collaboration [72, 73] which use the Bayesian method. Other groups obtain similar results.

In the standard unitarity triangle fit the results of the measurement of the following quantities are used

$$
\left|\frac{V_{u b}}{V_{c b}}\right|, \quad \Delta m_{d}, \frac{\Delta m_{d}}{\Delta m_{s}}, \epsilon \text { and } \sin 2 \beta
$$

From (120) we have

$$
\left|\frac{V_{u b}}{V_{c b}}\right|=\lambda \sqrt{\rho^{2}+\eta^{2}}=\frac{\lambda}{1-\frac{1}{2} \lambda^{2}} \sqrt{\bar{\rho}^{2}+\bar{\eta}^{2}},
$$

where $\bar{\rho}$ and $\bar{\eta}$ are determined by Eq. (126). Mass differences $\Delta m_{d}$ and $\Delta m_{s}$ are given by (105). For the ratio $\frac{\Delta m_{d}}{\Delta m_{s}}$ we have

$$
\frac{\Delta m_{d}}{\Delta m_{s}}=\lambda^{2}\left[(1-\bar{\rho})^{2}+\bar{\eta}^{2}\right] \frac{m_{B_{d}}}{m_{B_{s}}} \frac{f_{B_{d}}^{2} \hat{B}_{B_{d}}}{f_{B_{s}}^{2} \hat{B}_{B_{s}}}
$$

\footnotetext{
${ }^{14}$ The first error is statistical and second is systematical.
} 
The expression for the parameter $\epsilon$ is given by (237).

Let us obtain $\sin 2 \beta$ as a function of $\bar{\rho}$ and $\bar{\eta}$. From Fig. 1 we find that

$$
\sin \beta=\frac{\bar{\eta}}{\sqrt{(1-\bar{\rho})^{2}+\bar{\eta}^{2}}}, \quad \cos \beta=\frac{(1-\bar{\rho})}{\sqrt{(1-\bar{\rho})^{2}+\bar{\eta}^{2}}} .
$$

From these relations we have

$$
\sin 2 \beta=\frac{2 \bar{\eta}(1-\bar{\rho})}{(1-\bar{\rho})^{2}+\bar{\eta}^{2}} .
$$

From the fit of the experimental data the unique region in the plane of the parameters $\bar{\rho}, \bar{\eta}$ was found (see Fig 2).

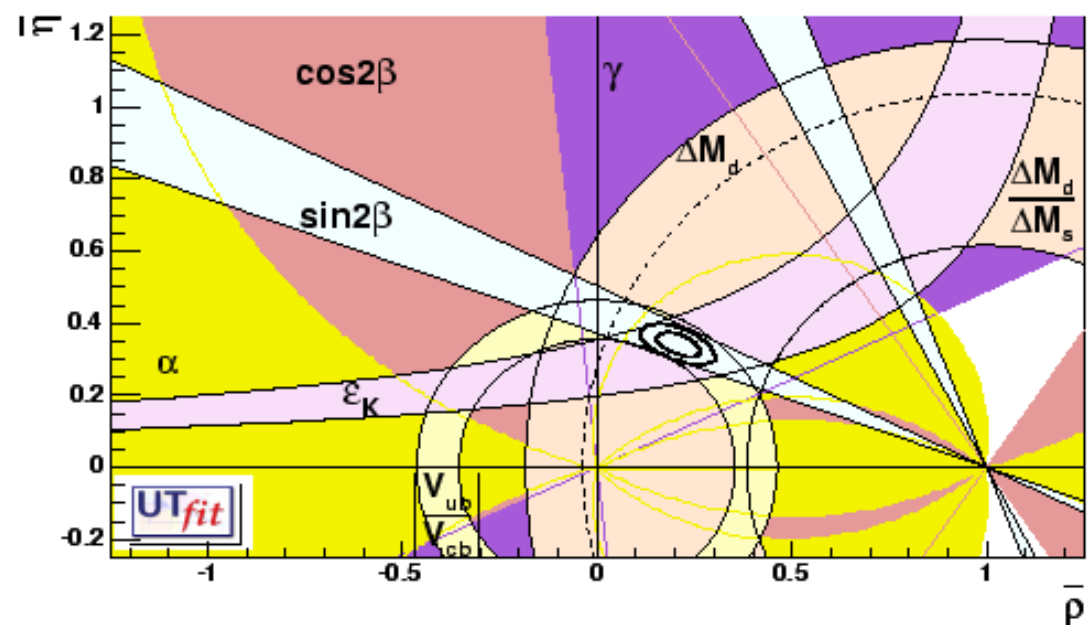

Figure 2: $68 \%$ and $95 \%$ total probability regions of the allowed values of the parameters $\bar{\rho}$ and $\bar{\eta}$. The values of the quantities $\left|V_{u b}\right| /\left|V_{c b}\right|, \Delta m_{d}, \Delta m_{s}, \epsilon$ and $\sin 2 \beta$ were used in the fit [72].

For the parameters $\bar{\rho}$ and $\bar{\eta}$ the following values were obtained

$$
\bar{\rho}=0.196 \pm 0.045, \quad \bar{\eta}=0.347 \pm 0.025 .
$$

These values determine the vertex B of the triangle in Fig 1.

The values of the parameters (289) overconstrain the unitarity triangle. For example, position of the vertex B can be obtained if only the parameters 
$\left|\frac{V_{u b}}{V_{c b}}\right|, \Delta m_{d}$ and $\Delta m_{s}$, which determine lengths of the sides of the triangle, are used in the fit. From the result of such fit the value of the parameter $\sin 2 \beta$ can be predicted. In 72 it was found

$$
\sin 2 \beta=0.734 \pm 0.043
$$

We can compare (295) with the measured values of the parameter $\sin 2 \beta$, given by (287) and (288). This comparison illustrates the evidence in favor of the correctness of the Standard Model.

Recently at the Tevatron in the Fermilab the mass difference $\Delta m_{s}$ was measured by D0 [61] and $C D F$ [59] collaborations. In the $C D F$ experiment it was found

$$
\Delta m_{s}=(17.77 \pm 0.10 \pm 0.07) p s^{-1} .
$$

The Belle collaboration measured the branching ratio for the leptonic decay $B \rightarrow \tau+\nu_{\tau}$ :

$$
B R\left(B_{d} \rightarrow \tau+\nu_{\tau}\right)=\left(1.06_{-0.28}^{0.34} \pm 0.18\right) \cdot 10^{-4} .
$$

From this measurement the value of the constant $f_{B_{d}}$ can be determined.

The BaBar and Belle Collaborations by the investigation of the decays $B_{d} \rightarrow \pi \pi, B_{d} \rightarrow \rho \rho, B_{d} \rightarrow \pi \pi \pi$ and $B_{d} \rightarrow D^{*} K^{*}$ obtained the information about the values of the angles $\alpha$ and $\gamma$ (see [76]).

In the new analysis of the UTfit collaboration [73] all these data were used. If in the analysis only the values of the angles $\alpha, \beta$ and $\gamma$ are used, for parameters $\bar{\rho}$ and $\bar{\eta}$ the following values were found

$$
\bar{\eta}=0.204 \pm 0.055, \quad \bar{\eta}=0.317 \pm 0.025 .
$$

If the quantities $\left|\frac{V_{u b}}{V_{c b}}\right|, \Delta m_{d}, \Delta m_{s}, \epsilon$ and results of the lattice calculations are used, in this case it was obtained

$$
\bar{\eta}=0.197 \pm 0.035, \quad \bar{\eta}=0.380 \pm 0.025 .
$$

From the fit of all data it was found

$$
\bar{\eta}=0.197 \pm 0.031, \quad \bar{\eta}=0.351 \pm 0.020 .
$$

The fit of all data is presented in Fig.3.

From this analysis (and analysis performed by other groups) we can conclude that existing data are in agreement with the Standard Model. However, it is necessary to stress that accuracy of the experimental data are limited and complicated QCD calculations are used in the analysis. There is still a room for beyond the SM physics (see,for example,[25]). In order to reveal it more precise data and progress in theoretical calculations are mandatory. 


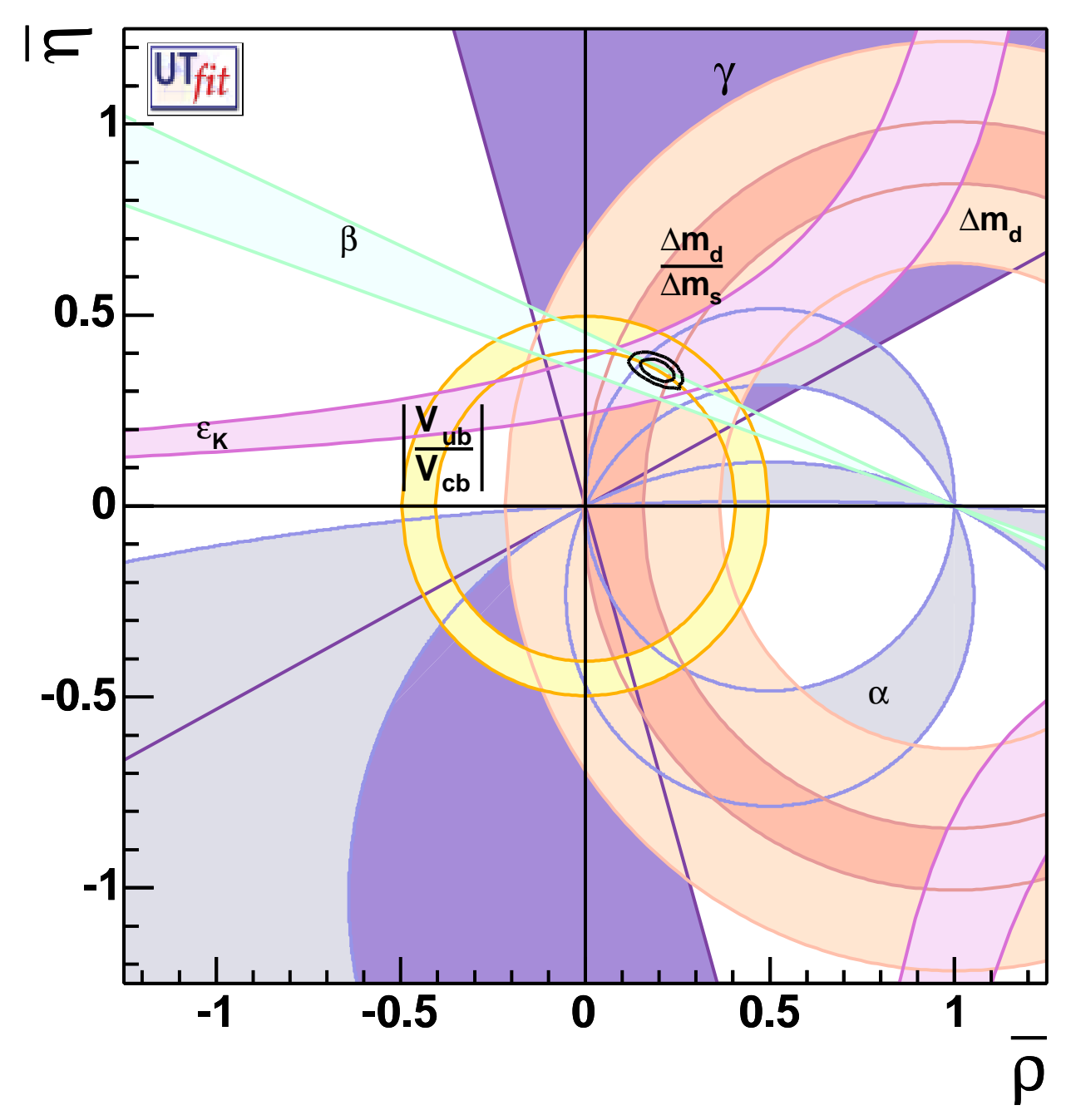

Figure 3: Allowed values of the parameters $\bar{\rho}$ and $\bar{\eta}$ (68\% and $95 \%$ total probability regions are shown [73]). The values of the quantities $\left|V_{u b}\right| /\left|V_{c b}\right|$, $\Delta m_{d}, \Delta m_{s}, \epsilon, \beta, \gamma$ and $\alpha$ were used in the fit . 


\section{Conclusion}

The Glashow-Weinberg-Salam unified theory of weak and electromagnetic interactions is outstanding achievement of the XX century physics. It was created as a result of a long period of the development of the phenomenological theory during which violation of $P, C$ [77] and later $C P$ [6] were discovered and universal $V-A$ current $\times$ current theory of the weak interaction were proposed [78, 79].

The SM predicted a new class of the weak interactions (neutral currents), vector $W^{ \pm}$and $Z^{0}$ bosons and their masses. After $\tau$-lepton was discovered the SM predicted that other members of the third family of quarks and leptons (b and t quarks and the third type of neutrino $\nu_{\tau}$ ) must exist. All predictions of the SM were perfectly confirmed by experiment.

Only one prediction of the SM, existence of the scalar neutral Higgs boson, is still waiting for its confirmation. The search for the Higgs boson will be one of the primary goal of the future LHC collider at CERN.

With the experiments at the LEP and SLD in the nineties next step in the testing of the SM started. The precision of these data required calculations of radiative corrections. At present numerous data of LEP, SLD, BaBar, Belle, CDF, D0 and other experiments are in a good agreement with the prediction of the SM. The fit of all electroweak data allows to predict the upper bound of the mass of the Higgs boson (see, for example, [32]): $m_{H} \leq 235 \mathrm{GeV}$ at 99 $\%$ CL.

In 1999 with the beginning of BaBar and Belle experiments at asymmetrical B-factories at SLAC and KEK a new stage in the testing of the SM started. In the framework of the SM violation of the $C P$ invariance is due to one physical phase in the unitary $3 \times 3 C K M$ mixing matrix. This phase enter into the unitarity triangle relation which is a consequence of the orthogonality of different columns (or lines) of the mixing matrix. The numerous tests of this relation became possible with new B-factory data. Existing data are in a good agreement with the Standard Model. This agreement confirms

- The basic assumption of the SM that two sets of quarks fields, fields $q_{L}^{\prime}$ which possess definite transformation property and fields of quarks with definite masses $q_{L}$, are connected by unitary transformation.

- The assumption that only three quarks families exist in nature.

It is necessary, however, to stress that the unitarity triangle test is based not only on experimental data but also on nonperturbative $Q C D$ calculations of 
relevant matrix elements. A room for a new physics is still open. Improvement of the accuracy of data and improvement of the accuracy of the lattice and other calculations is a key problem for the future progress.

It is a pleasure for me to acknowledge the ILIAS program for the support and theory department of the TRIUMF for the hospitality.

\section{Appendix A. Comparison of $M^{0} \leftrightarrows \bar{M}^{0}$ oscillations with neutrino oscillations}

It is of interest to compare $M^{0} \leftrightarrows \bar{M}^{0}$ oscillations with neutrino oscillations recently discovered in the Super Kamiokande [80], SNO [81], KamLAND [82] and other neutrino oscillation experiments [83, 84, 85, 87, 88].

Particles with definite flavor $M^{0}$ and $\bar{M}^{0}\left(M^{0}=K^{0}, B_{d, s}^{0}, \ldots\right)$ are produced in strong interaction processes. States of these particles are eigenstates of the Hamiltonian of the strong and electromagnetic interactions which conserve the quark flavor. In processes of production of $M^{0}$ and $\bar{M}^{0}$ effects of the weak interaction, in which quark flavor is violated, are negligibly small and can be neglected. After $M^{0}$ and $\bar{M}^{0}$ are produced weak interaction play the major role. Because of the weak interaction $M^{0}$ and $\bar{M}^{0}$ decay and eigenstates of the total Hamiltonian $\left|M_{H}^{0}\right\rangle$ and $\left|\bar{M}_{L}^{0}\right\rangle$ have different masses and widths. Thus, $M^{0} \leftrightarrows \bar{M}^{0}$ oscillations are due to the existence of strong interaction in which quark flavor is conserved and weak interaction in which quark flavor is changed.

Neutrinos have only weak interaction. However, neutrino masses are very small. In neutrino production and neutrino detection processes neutrino masses can be safely neglected. This means that in such processes lepton flavor numbers $L_{e}, L_{\mu}$ and $L_{\tau}$ are conserved: together with lepton $l^{+}$flavor neutrino $\nu_{l}$ is produced, flavor neutrino $\nu_{l}$ in a charge current process produce $l^{-}$etc . After flavor neutrino is produced small neutrino masses (and neutrino mixing) play the key role. Because of neutrino masses, in the neutrino propagation different mass components of the mixed flavor neutrino state acquire different phases. This is a physical reason for the neutrino oscillations $\nu_{l} \leftrightarrows \nu_{l^{\prime}}$ (see, for example, [89, 90])

In the framework of the Standard Model $C P$ violation is connected with the physical phase in the mixing matrix. As we have seen, in the case of two families there are no physical phases in the mixing matrix. The $C P$ invariance can be violated if (at least) three families of the quark and leptons exist. 
In the case of neutral bosons oscillations take place only between two particles $M^{0}$ and $\bar{M}^{0}$. In order to reveal $C P$ violation in decays of these particles one must observe effects of all three families of quarks. Because observable particles are hadrons and not quarks Jarlskog invariant $J$ does not enter in the quantities which characterize the $C P$ violation.

Neutrinos are stable particles. In order to observe effects of the $C P$ violation in neutrino oscillations all three neutrinos must be involved in the transition probability. Only elements of the neutrino mixing matrix and the neutrino mass-squared differences enter into the transition probabilities. It is natural to expect that effects of the $C P$ violation in the neutrino oscillations are determined by the Jarlskog invariant. We will demonstrate this below.

The Lagrangian of neutrino interaction has the form

$$
\mathcal{L}^{C C}(x)=-\frac{g}{2 \sqrt{2}} j_{\alpha}^{C C}(x) W^{\alpha}(x)+\text { h.c., }, \quad \mathcal{L}^{\mathrm{NC}}(\mathrm{x})=-\frac{\mathrm{g}}{2 \cos \theta_{\mathrm{W}}} \mathrm{j}_{\alpha}^{\mathrm{NC}}(\mathrm{x}) \mathrm{Z}^{\alpha}(\mathrm{x}),
$$

where the charged current $j_{\alpha}^{C C}(x)$ and the neutral current $j_{\alpha}^{N C}(x)$ are given by the expressions

$$
j_{\alpha}^{\mathrm{CC}}(x)=2 \sum_{l=e, \mu, \tau} \bar{\nu}_{l L}(x) \gamma_{\alpha} l_{L}(x), \quad j_{\alpha}^{\mathrm{NC}}(x)=\sum_{l=e, \mu, \tau} \bar{\nu}_{l L}(x) \gamma_{\alpha} \nu_{l L}(x) .
$$

Here

$$
\nu_{l L}(x)=\sum_{k=1}^{3} U_{l i} \nu_{i L}(x),
$$

is the "mixed field". In (A-3) $\nu_{i}(x)$ is the field of neutrino with mass $m_{i}$ and $U$ is the unitary $3 \times 3$ PMNS mixing matrix [91, 92]. For neutrinos, particles with electric charges equal to zero, there are two possibilities (see [93, 94])

1. If the total lepton number $L=L_{e}+L_{\mu}+L_{\tau}$ is conserved, neutrinos $\nu_{i}$ are Dirac particles.

2. If there are no conserved lepton numbers, neutrinos $\nu_{i}$ are Majorana particles.

The probabilities of the transition $\nu_{l} \rightarrow \nu_{l^{\prime}}$ and $\nu_{l} \rightarrow \nu_{l^{\prime}}\left(l, l^{\prime}=e, \mu, \tau\right)$ during the time $t$ in the three-neutrino case, we are considering, is given by the following expressions (see, for example, 94])

$$
P\left(\nu_{l} \rightarrow \nu_{l^{\prime}}\right)=\left|\sum_{i=1}^{3} U_{l^{\prime} i} e^{-i E_{i} t} U_{l i}^{*}\right|^{2}
$$


and

$$
P\left(\bar{\nu}_{l} \rightarrow \bar{\nu}_{l^{\prime}}\right)=\left|\sum_{i=1}^{3} U_{l^{\prime} i}^{*} e^{-i E_{i} t} U_{l i}\right|^{2} .
$$

Here $E_{i} \simeq p+\frac{m_{i}^{2}}{2 p}$ is the energy of neutrino with mass $m_{i}$ and momentum $p$.

If $C P$ is conserved in the case of the Dirac neutrinos arbitrary phases of the fields of the leptons and neutrinos can be chosen in such a way that

$$
U_{l i}^{*}=U_{l i}
$$

If $\nu_{i}$ are Majorana particles from the condition of the $C P$ invariance we have (see [95])

$$
U_{l i}^{*}=U_{l i} \eta_{i}
$$

where $\eta_{i}= \pm i$ is the $C P$-parity of the Majorana neutrino with the mass $m_{i}$.

From (A-4), (A-5), (A-6) and (A-7) it follows that in the case of the $C P$ invariance for the Dirac as well as for the Majorana neutrinos we have

$$
P\left(\nu_{l} \rightarrow \nu_{l^{\prime}}\right)=P\left(\bar{\nu}_{l} \rightarrow \bar{\nu}_{l^{\prime}}\right)
$$

If we compare expressions $(\underline{A-4})$ and $(\mathrm{A}-5)$ we come to the conclusion that transition probabilities satisfy the following relation

$$
P\left(\nu_{l} \rightarrow \nu_{l^{\prime}}\right)=P\left(\bar{\nu}_{l^{\prime}} \rightarrow \bar{\nu}_{l}\right) .
$$

This relation is the consequence of the $C P T$ invariance inherent to any local quantum field theory. It follows from (A-9) that the equality

$$
P\left(\nu_{l} \rightarrow \nu_{l}\right)=P\left(\bar{\nu}_{l} \rightarrow \bar{\nu}_{l}\right)
$$

is a consequence of the $C P T$ invariance. Thus, if the inequality

$$
P\left(\nu_{l} \rightarrow \nu_{l^{\prime}}\right) \neq P\left(\bar{\nu}_{l^{\prime}} \rightarrow \bar{\nu}_{l}\right), \quad l^{\prime} \neq l
$$

takes place it would be a proof of the $C P$ violation in the lepton sector.

Let us consider now the expression (A-4) for the transition probability $P\left(\nu_{l} \rightarrow \nu_{l^{\prime}}\right)$. We have

$$
P\left(\nu_{l} \rightarrow \nu_{l^{\prime}}\right)=\sum_{i, k} U_{l^{\prime} i} U_{l^{\prime} k}^{*} U_{l i}^{*} U_{l k} e^{-i\left(E_{i}-E_{k}\right) t} .
$$


Here

$$
\left(E_{i}-E_{k}\right) t \simeq \frac{\Delta m_{k i}^{2}}{2 E} L
$$

where $L$ is the distance between the neutrino production and the neutrino detection points and $\Delta m_{k i}^{2}=m_{i}^{2}-m_{k}^{2}$. From (A-12) for the transition probability we find the following expression

$$
P\left(\nu_{l} \rightarrow \nu_{l^{\prime}}\right)=\sum_{i}\left|U_{l^{\prime} i}\right|^{2}\left|U_{l i}\right|^{2}+2 \operatorname{Re} \sum_{\mathrm{i}>\mathrm{k}} \mathrm{U}_{\mathrm{l}^{\prime} \mathrm{i}} \mathrm{U}_{\mathrm{l}^{\prime} \mathrm{k}}^{*} \mathrm{U}_{\mathrm{li}}^{*} \mathrm{U}_{\mathrm{lk}} \mathrm{e}^{-\mathrm{i} \frac{\Delta \mathrm{m}_{\mathrm{ki}}^{2}}{2 \mathrm{E}} \mathrm{L}} .
$$

Further, from the condition of the unitarity of the mixing matrix $U$

$$
\sum_{i} U_{l^{\prime} i} U_{l i}^{*}=\delta_{l^{\prime} l}
$$

we find

$$
\sum_{i}\left|U_{l^{\prime} i}\right|^{2}\left|U_{l i}\right|^{2}=\delta_{l^{\prime} l}-2 \operatorname{Re} \sum_{\mathrm{i}>\mathrm{k}} \mathrm{U}_{\mathrm{l}^{\prime} \mathrm{i}} \mathrm{U}_{\mathrm{l}^{\prime} \mathrm{k}}^{*} \mathrm{U}_{\mathrm{li}}^{*} \mathrm{U}_{\mathrm{lk}}
$$

From (A-14) and (A-16) for the transition probability we obtain the following expression

$$
P\left(\nu_{l} \rightarrow \nu_{l^{\prime}}\right)=\delta_{l^{\prime} l}-2 \operatorname{Re} \sum_{\mathrm{i}>\mathrm{k}} \mathrm{U}_{\mathrm{l}^{\prime} \mathrm{i}} \mathrm{U}_{\mathrm{l}^{\prime} \mathrm{k}}^{*} \mathrm{U}_{\mathrm{li}}^{*} \mathrm{U}_{\mathrm{lk}}\left(1-\mathrm{e}^{-\mathrm{i} \frac{\Delta \mathrm{m}_{\mathrm{ki}}^{2}}{2 \mathrm{E}} \mathrm{L}}\right) .
$$

From (A-17) we have

$$
\begin{aligned}
P\left(\nu_{l} \rightarrow \nu_{l^{\prime}}\right) & =\delta_{l^{\prime} l}-2 \sum_{i>k} \operatorname{Re}\left(\mathrm{U}_{\mathrm{l}^{\prime} \mathrm{i}} \mathrm{U}_{\mathrm{l}^{\prime} \mathrm{k}}^{*} \mathrm{U}_{\mathrm{li}}^{*} \mathrm{U}_{\mathrm{lk}}\right)\left(1-\cos \frac{\Delta \mathrm{m}_{\mathrm{ki}}^{2}}{2 \mathrm{E}} \mathrm{L}\right) \\
& +2 \sum_{i>k} \operatorname{Im}\left(\mathrm{U}_{\mathrm{l}^{\prime} \mathrm{i}} \mathrm{U}_{\mathrm{l}^{\prime} \mathrm{k}}^{*} \mathrm{U}_{\mathrm{li}}^{*} \mathrm{U}_{\mathrm{lk}}\right) \sin \frac{\Delta \mathrm{m}_{\mathrm{ki}}^{2} \mathrm{~L}}{2 \mathrm{E}}
\end{aligned}
$$

Analogously, for the probability of the transition $\bar{\nu}_{l} \rightarrow \bar{\nu}_{l^{\prime}}$ we find

$$
\begin{aligned}
P\left(\bar{\nu}_{l} \rightarrow \bar{\nu}_{l^{\prime}}\right) & =\delta_{l^{\prime} l}-2 \sum_{i>k} \operatorname{Re}\left(\mathrm{U}_{\mathrm{l}^{\prime} \mathrm{i}} \mathrm{U}_{\mathrm{l}^{\prime} \mathrm{k}}^{*} \mathrm{U}_{\mathrm{li}}^{*} \mathrm{U}_{\mathrm{lk}}\right)\left(1-\cos \frac{\Delta \mathrm{m}_{\mathrm{ki}}^{2}}{2 \mathrm{E}} \mathrm{L}\right) \\
& -2 \sum_{i>k} \operatorname{Im}\left(\mathrm{U}_{\mathrm{l}^{\prime} \mathrm{i}} \mathrm{U}_{\mathrm{l}^{\prime} \mathrm{k}}^{*} \mathrm{U}_{\mathrm{li}}^{*} \mathrm{U}_{\mathrm{lk}}\right) \sin \frac{\Delta \mathrm{m}_{\mathrm{ki}}^{2} \mathrm{~L}}{2 \mathrm{E}}
\end{aligned}
$$


Let us introduce the quantity

$$
J_{l^{\prime} l}^{i k}=\operatorname{Im} \mathrm{U}_{\mathrm{l}^{\prime} \mathrm{i}} \mathrm{U}_{\mathrm{lk}} \mathrm{U}_{\mathrm{l}^{\prime} \mathrm{k}}^{*} \mathrm{U}_{\mathrm{li}}^{*} \text {. }
$$

In the case of the $C P$ invariance from $(\underline{\mathrm{A}-6})$ for the Dirac neutrinos and from (A-7) for the Majorana neutrinos we find

$$
J_{l^{\prime} l}^{i k}=0 .
$$

From the definition (A-20) we have

$$
J_{l^{\prime} l}^{i k}=-J_{l^{\prime} l}^{k i}, \quad J_{l^{\prime} l}^{i k}=-J_{l l^{\prime}}^{i k} .
$$

Further, from the unitarity of the $3 \times 3$ mixing matrix $U$ we find

$$
\sum_{i} J_{l^{\prime} l}^{i k}=\delta_{l^{\prime} l} \operatorname{Im} \mathrm{U}_{\mathrm{l}^{\prime} \mathrm{k}}^{*} \mathrm{U}_{\mathrm{lk}}=0, \quad \sum_{\mathrm{l}^{\prime}} \mathrm{J}_{\mathrm{l}^{\prime} \mathrm{l}}^{\mathrm{ik}}=\delta_{\mathrm{ik}} \operatorname{Im} \mathrm{U}_{\mathrm{li}}^{*} \mathrm{U}_{\mathrm{lk}}=0 .
$$

From (A-22) and the first equation (A-23) we have

$$
J_{l^{\prime} l}^{21}=J_{l^{\prime} l}^{13}=J_{l^{\prime} l}^{32} .
$$

Further, from $(\underline{\mathrm{A}-22})$ and the second equation $(\mathrm{A}-23)$ we find

$$
J_{e \mu}^{i k}=J_{\mu \tau}^{i k}=J_{\tau e}^{i k} .
$$

If we introduce the following notation

$$
J_{e \mu}^{21}=J
$$

from (A-24) and (A-25) we have

$$
J_{l^{\prime} l}^{i k}= \pm J, \quad l^{\prime} \neq l, \quad i \neq k .
$$

Thus, in the neutrino case, as in the quark case (see section 3), exist only one independent Jarlskog invariant.

Let us consider now the last term of the expression (A-18) for $l^{\prime} \neq l$. Taking into account ( $(\mathrm{A}-22)$, we find

$$
2 \sum_{i>k} J_{l^{\prime} l}^{i k} \sin \frac{\Delta m_{k i}^{2}}{2 E} L=2 J_{l^{\prime} l}^{21}\left(\sin \frac{\Delta m_{12}^{2}}{2 E} L+\sin \frac{\Delta m_{23}^{2}}{2 E} L-\sin \frac{\Delta m_{13}^{2}}{2 E} L\right) .
$$


It is obvious that

$$
\Delta m_{13}^{2}=\Delta m_{12}^{2}+\Delta m_{23}^{2} .
$$

Further, for any $a$ and $b$ we have

$$
\sin a+\sin b-\sin (a+b)=4 \sin \frac{a}{2} \sin \frac{b}{2} \sin \frac{(a+b)}{2}
$$

From (A-28), (A-29) and (A-30) we find

$$
2 \sum_{i>k} J_{l^{\prime} l}^{i k} \sin \frac{\Delta m_{k i}^{2}}{2 E} L=8 J_{l^{\prime} l}^{21} \sin \frac{\Delta m_{12}^{2}}{4 E} L \sin \frac{\Delta m_{23}^{2}}{4 E} L \sin \frac{\left(\Delta m_{12}^{2}+\Delta m_{23}^{2}\right)}{4 E} L .
$$

Let us determine $C P$ asymmetry

$$
A_{l^{\prime} l}^{C P}=P\left(\nu_{l} \rightarrow \nu_{l^{\prime}}\right)-P\left(\bar{\nu}_{l} \rightarrow \bar{\nu}_{l^{\prime}}\right) \quad l \neq l^{\prime}
$$

From the unitarity of the mixing matrix and the CPT invariance it easy to obtain the following relations

$$
A_{e \mu}^{C P}=A_{\tau e}^{C P}=-A_{\tau \mu}^{C P} .
$$

In fact, from the unitarity of the mixing matrix we find

$$
\sum_{l^{\prime}=e, \mu, \tau} A_{l^{\prime} l}^{C P}=0
$$

Further, from the relation (A-9) we have

$$
A_{l^{\prime} l}^{C P}=-A_{l l^{\prime}}^{C P} .
$$

From (A-34) and (A-35) we obtain the following relations

$$
A_{\mu e}^{C P}+A_{\tau e}^{C P}=0, A_{e \mu}^{C P}+A_{\tau \mu}^{C P}=0, A_{e \tau}^{C P}+A_{\mu \tau}^{C P}=0 .
$$

From (A-36) we easily find the relations (A-33). Thus, in the case of three families exist only one independent asymmetry.

From (A-18), (A-19), (A-31) and (A-32) we find

$$
A_{e \mu}^{C P}=16 J \sin \frac{\Delta m_{12}^{2}}{4 E} L \sin \frac{\Delta m_{23}^{2}}{4 E} L \sin \frac{\left(\Delta m_{12}^{2}+\Delta m_{23}^{2}\right)}{4 E} L .
$$


Thus, the $C P$ asymmetry is proportional to the invariant $J$. Let us comment this connection.

The transition probabilities $(\underline{\mathrm{A}-4})$ and $(\underline{\mathrm{A}-5})$ are invariant under the phase transformation

$$
U_{l i} \rightarrow e^{-i \alpha_{l}} U_{l i} e^{-i \beta_{i}},
$$

where $\alpha_{l}$ and $\beta_{i}$ are arbitrary constant phases. It is obvious that

1. The $C P$ asymmetry is also invariant under the transformation (A-38).

2. The $C P$ asymmetry is equal to zero in the case of the $C P$ invariance.

The Jarlskog invariant $J$ satisfies both these conditions.

As we have seen there exists only one Jarlskog invariant in the case of the three-neutrino mixing. This is connected with the fact that only one phase characterizes the mixing matrix. Let us comment now this last statement. In the case of the Dirac neutrinos, like in the quark case, one physical $C P$ phase characterizes mixing matrix. In the case of the Majorana neutrinos $3 \times 3$ mixing matrix is characterized by three $C P$ phases 96 . However, two additional Majorana phases do not enter into expressions for neutrino and antineutrino transition probabilities [96].

The $3 \times 3 P M N S$ neutrino mixing matrix can be parameterized in the same way as $C K M$ quark mixing matrix (see (78)). For the Jarlskog invariant we have in this case

$$
J=-s_{12} s_{13} s_{23} \sin \delta c_{13}^{2} c_{12} c_{23}
$$

It follows from (A-37) and (A-39) that in order the $C P$ asymmetry is different from zero it is necessary that not only the $C P$ phase but also three mixing angles $\theta_{12}, \theta_{23}$ and $\theta_{13}$ and two mass-squared differences $\Delta m_{23}^{2}$ and $\Delta m_{12}^{2}$ are different from zero. Thus, in order to reveal violation of the $C P$ invariance in the lepton sector all three families must be involved in oscillations.

\section{Appendix B. Evolution equation for $M^{0}-\bar{M}^{0}$ system}

The physics of the $M^{0}-\bar{M}^{0}$ system $\left(M^{0}=K^{0}, B_{d, s}^{0}, \ldots\right)$ is based on the evolution equation. We will show here that wave functions of such systems satisfy the Schrodinger equation with non hermitian Hamiltonian.

Let us consider, as an example, $K^{0}-\bar{K}^{0}$ system. $K^{0}$ and $\bar{K}^{0}$ mesons, are particles with the strangeness +1 and -1 , correspondingly. They are 
produced in strong interaction processes in which strangeness is conserved. After $K^{0}\left(\bar{K}^{0}\right)$ is produced weak interaction in which strangeness is changed plays the major role: due to weak interaction particles decay and transitions $K^{0} \leftrightarrows \bar{K}^{0}$ take place

We will present the total Hamiltonian in the form

$$
H=H_{0}+H_{W}
$$

where $H_{0}$ is a sum of the free Hamiltonian and the Hamiltonian of strong and electromagnetic interactions and $H_{W}$ is the Hamiltonian of the weak interaction.

Let $\left|K^{0}\right\rangle$ and $\left|\bar{K}^{0}\right\rangle$ be the states of $K^{0}$ and $\bar{K}^{0}$ in their rest systems. These states are eigenstates of the Hamiltonian $H_{0}$ and of the operator of the strangeness S. Assuming CPT invariance of the Hamiltonian of the strong and electromagnetic interactions we have

$$
H_{0}\left|K^{0}\right\rangle=m\left|K^{0}\right\rangle, \quad H_{0}\left|\bar{K}^{0}\right\rangle=m\left|\bar{K}^{0}\right\rangle
$$

and

$$
S\left|K^{0}\right\rangle=\left|K^{0}\right\rangle, \quad S\left|\bar{K}^{0}\right\rangle=-\left|\bar{K}^{0}\right\rangle,
$$

where $m$ is the mass. Due to the $C P T$ invariance the masses of $K^{0}$ and $\bar{K}^{0}$ are the same.

Because of the conservation of the strangeness by the Hamiltonian $H_{0}$ the vectors $\left|K^{0}\right\rangle$ and $\left.\overline{\mid} K^{0}\right\rangle$ can not be distinguished from vectors

$$
\left|K^{0}\right\rangle^{\prime}=e^{i S \alpha}\left|K^{0}\right\rangle=e^{i \alpha}\left|K^{0}\right\rangle, \quad\left|\bar{K}^{0}\right\rangle^{\prime}=e^{i S \alpha}\left|\bar{K}^{0}\right\rangle=e^{-i \alpha}\left|\bar{K}^{0}\right\rangle,
$$

where $\alpha$ is an arbitrary constant phase.

The operators of the $C P$ conjugation and the strangeness anticommute with each other

$$
C P S+S C P=0
$$

From this relation we have

$$
S C P\left|K^{0}\right\rangle=-C P\left|K^{0}\right\rangle \text {. }
$$

Thus, we have

$$
C P\left|K^{0}\right\rangle=\eta_{C P}\left|\bar{K}^{0}\right\rangle,
$$


where $\eta_{C P}$ is a $C P$ phase factor. Taking into account the freedom in the choice of the phases of the vectors $\left|K^{0}\right\rangle$ and $\left|\bar{K}^{0}\right\rangle$ we can put $\eta_{C P}=1$. In this case we have

$$
C P\left|K^{0}\right\rangle=\left|\bar{K}^{0}\right\rangle .
$$

In this review we used this choice. However, we demonstrated that measurable quantities does not depend on the choice of arbitrary phase factors.

Let us consider now the Schrodinger equation for a vector $|\Psi(t)\rangle$. We have

$$
i \frac{\partial|\Psi(t)\rangle}{\partial t}=H|\Psi(t)\rangle .
$$

The formal general solution of the equation (B-9) has the form

$$
|\Psi(t)\rangle=e^{i H t}|\Psi(0)\rangle,
$$

where $|\Psi(0)\rangle$ is the vector of the state at the initial time $t=0$.

It will be convenient to present the solution $(\underline{B-10})$ in another form. Let us denote $|n\rangle$ the normalized eigenvector of the total Hamiltonian. We have

$$
H|n\rangle=E_{n}|n\rangle, \quad\left\langle n^{\prime} \mid n\right\rangle=\delta_{n^{\prime} n} .
$$

The vector $|\Psi(0)\rangle$ can be developed over the vectors $|n\rangle$. We have

$$
|\Psi(0)\rangle=\sum_{n}|n\rangle\langle n \mid \Psi(0)\rangle
$$

From (B-10), (B-11) and $(\underline{B-12})$ we find

$$
|\Psi(t)\rangle=\sum_{n} e^{-i E_{n} t}|n\rangle\langle n \mid \Psi(0)\rangle
$$

Further for $t \geq 0$ we have

$$
e^{-i E_{n} t}=\frac{-1}{2 \pi i} \int_{-\infty}^{\infty} \frac{e^{-i E t}}{E-E_{n}+i \epsilon} d E
$$

From (B-11), (B-13) and $(\overline{B-14})$ we find that the solution of the equation (B-9) can be presented in the form

$$
|\Psi(t)\rangle=\frac{-1}{2 \pi i} \int_{-\infty}^{\infty} G_{+}(E) e^{-i E t} d E|\Psi(0)\rangle
$$


where

$$
G_{+}(E)=\frac{1}{E-H+i \epsilon}
$$

We assume now that the initial state $|\Psi(0)\rangle$ is a superposition of the states of $K^{0}$ and $\bar{K}^{0}$ mesons. We have

$$
|\Psi(0)\rangle=\sum_{\alpha=1,2} a_{\alpha}(0)|\alpha\rangle,
$$

where $\left|K^{0}\right\rangle \equiv|1\rangle$ and $\left|\bar{K}^{0}\right\rangle \equiv|2\rangle$.

At $t \geq 0$ we have

$$
|\Psi(t)\rangle=\sum_{\alpha=1,2} a_{\alpha}(t)|\alpha\rangle+\sum_{i} b_{i}(t)|i\rangle,
$$

where $a_{1}(t)\left(a_{2}(t)\right)$ is the amplitude of the probability to find $K^{0}\left(\bar{K}^{0}\right)$ at time $t$ and $|i\rangle$ are states of the particles which are produced in decays of neutral kaons $\left(\pi \pi, \pi \pi \pi, \pi l \nu_{l}\right.$ etc $)$.

From (B-15) and (B-17) for the wave function $a_{\alpha}(t)$ we find the following expression

$$
a_{\alpha^{\prime}}(t)=\left\langle\alpha^{\prime} \mid \Psi(t)\right\rangle=\frac{-1}{2 \pi i} \int_{-\infty}^{\infty} \sum_{\alpha}\left\langle\alpha^{\prime}\left|G_{+}(E)\right| \alpha\right\rangle e^{-i E t} d E a_{\alpha}(0)
$$

Up to now all our equations were exact. Now we will develop perturbation theory over the weak interaction. From $(\underline{\mathrm{B}-16})$ we have

$$
\left(E-H_{0}-H_{W}+i \epsilon\right) G_{+}(E)=1
$$

If we multiply this equation by the operator $\frac{1}{E-H_{0}+i \epsilon}$ from the left we obtain the Lippman-Schwinger equation for the operator $G_{+}(E)$ :

$$
G_{+}(E)=\frac{1}{E-H_{0}+i \epsilon}+\frac{1}{E-H_{0}+i \epsilon} H_{W} G_{+}(E) .
$$

We will obtain now the matrix element $\left\langle\alpha^{\prime}\left|G_{+}(E)\right| \alpha\right\rangle$ in the form of perturbation series. From (B-2) we find 15

$$
\begin{aligned}
\left\langle\alpha^{\prime}\left|G_{+}(E)\right| \alpha\right\rangle= & \frac{\delta_{\alpha^{\prime} \alpha}}{E-m+i \epsilon}+\frac{1}{E-m+i \epsilon} \sum_{\alpha^{\prime \prime}}\left\langle\alpha^{\prime}\left|H_{W}\right| \alpha^{\prime \prime}\right\rangle\left\langle\alpha^{\prime \prime}\left|G_{+}(E)\right| \alpha\right\rangle+ \\
& +\frac{1}{E-m+i \epsilon} \sum_{i}\left\langle\alpha^{\prime}\left|H_{W}\right| i\right\rangle\left\langle i\left|G_{+}(E)\right| \alpha\right\rangle .
\end{aligned}
$$

\footnotetext{
${ }^{15}$ The sum $\sum_{i}$ means sum and integration over corresponding variables in the state $|i\rangle$ and sum over all possible states $|i\rangle$.
} 
Now, taking into account that $\langle i \mid \alpha\rangle=0$ from (B-21) we find

$$
\begin{aligned}
\left\langle i\left|G_{+}(E)\right| \alpha\right\rangle= & \frac{1}{E-E_{i}+i \epsilon}\left[\sum_{\alpha^{\prime \prime}}\left\langle i\left|H_{W}\right| \alpha^{\prime \prime}\right\rangle\left\langle\alpha^{\prime \prime}\left|G_{+}(E)\right| \alpha\right\rangle+\right. \\
& \left.+\sum_{i^{\prime}}\left\langle i\left|H_{W}\right| i^{\prime}\right\rangle\left\langle i^{\prime}\left|G_{+}(E)\right| \alpha\right\rangle\right]
\end{aligned}
$$

This equation can be easily solved by iterations. Its solution can be presented in form of the perturbation series over the weak interaction. We will consider only the first term of the series.

From (B-22) and (B-23) we find

$\left\langle\alpha^{\prime}\left|G_{+}(E)\right| \alpha\right\rangle=\frac{\delta_{\alpha^{\prime} \alpha}}{E-m+i \epsilon}+\frac{1}{E-m+i \epsilon} \sum_{\alpha_{1}}\left\langle\alpha^{\prime}|R(E)| \alpha^{\prime \prime}\right\rangle\left\langle\alpha^{\prime \prime}\left|G_{+}(E)\right| \alpha\right\rangle$,

where up to the terms of the second order of the perturbation theory we have $\left\langle\alpha^{\prime}|R(E)| \alpha^{\prime \prime}\right\rangle=\left\langle\alpha^{\prime}\left|H_{W}\right| \alpha^{\prime \prime}\right\rangle+\sum_{i}\left\langle\alpha^{\prime}\left|H_{W}\right| i\right\rangle \frac{1}{E-E_{i}+i \epsilon}\left\langle i\left|H_{W}\right| \alpha^{\prime \prime}\right\rangle+\ldots$

In the matrix form the equation $(\overline{\mathrm{B}-24})$ can be written as follows

$$
G_{+}(E)=\frac{1}{E-m+i \epsilon}+\frac{1}{E-m+i \epsilon} R(E) G_{+}(E),
$$

where $G_{+}(E)$ and $R(E)$ are $2 \times 2$ matrices with elements $\left\langle\alpha^{\prime}\left|G_{+}(E)\right| \alpha\right\rangle$ and $\left\langle\alpha^{\prime}|R(E)| \alpha\right\rangle$. This matrix equation can be easily solved. We have

$$
G_{+}(E)=\frac{1}{E-m-R(E)+i \epsilon}
$$

From (B-19) and (B-27) for the wave function $a(t)$ we find

$$
a(t)=\frac{-1}{2 \pi i} \int_{-\infty}^{\infty} \frac{e^{-i E t}}{E-m-R(E)+i \epsilon} d E a(0)
$$

Because $|R(E)| \ll m$ the pole in the integral $(\overline{\mathrm{B}-28})$ is at the point $E \simeq$ $m$. We have $R(E)=R(m)+\left.(E-m) \frac{d R}{d E}\right|_{E=m}+\ldots$. The second term of this expansion is much smaller than the first one. We will neglect it. This 
approximation is called the Weisskopf-Wigner approximation [97]. In this approximation we have 16

$$
a(t) \simeq \frac{-1}{2 \pi i} \int_{-\infty}^{\infty} \frac{e^{-i E t}}{E-\mathcal{H}+i \epsilon} d E a(0)=e^{-i \mathcal{H} t} a(0),
$$

where

$$
\mathcal{H}=m+R(m)
$$

From (B-29) we come to the conclusion that the wave function of $K^{0}-\bar{K}^{0}$ system satisfies the Schrodinger equation

$$
i \frac{\partial a(t)}{\partial t}=\mathcal{H} a(t)
$$

Let us consider now the effective Hamiltonian $\mathcal{H}$. Taking into account the relation

$$
\frac{1}{m-E_{i}+i \epsilon}=P \frac{1}{m-E_{i}}-i \pi \delta\left(E_{i}-m\right)
$$

from (B-25) we find

$$
\mathcal{H}=M-\frac{i}{2} \Gamma
$$

where

$$
M_{\alpha^{\prime} \alpha}=m \delta_{\alpha^{\prime} \alpha}+\left\langle\alpha^{\prime}\left|H_{W}\right| \alpha\right\rangle+P \sum_{i}\left\langle\alpha^{\prime}\left|H_{W}\right| i\right\rangle \frac{1}{m-E_{i}}\left\langle i\left|H_{W}\right| \alpha\right\rangle
$$

and

$$
\Gamma_{\alpha^{\prime} \alpha}=2 \pi \sum_{i}\left\langle\alpha^{\prime}\left|H_{W}\right| i\right\rangle\left\langle i\left|H_{W}\right| \alpha\right\rangle \delta\left(E_{i}-m\right)
$$

It follows from these expressions that $M$ and $\Gamma$ are hermitian matrices:

$$
M^{\dagger}=M, \quad \Gamma^{\dagger}=\Gamma .
$$

Thus, summarizing, wave function of $K^{0}-\bar{K}^{0}$ system satisfies the Schrodinger equation with effective non hermitian Hamiltonian $\mathcal{H}$ which is given by (B-33).

Let us consider $M_{11}\left(M_{22}\right)$. The first term in (B-34) is the bare mass of $K^{0}\left(\bar{K}^{0}\right)$. The second and third terms are the corrections to mass. Thus, $M_{11}$

\footnotetext{
${ }^{16}$ We took into account that imaginary parts of the eigenvalues of $\mathcal{H}$ are negative
} 
$\left(M_{22}\right)$ is the mass of $K^{0}\left(\bar{K}^{0}\right)$ with corrections due to the weak interaction. From the $C P T$ invariance it follows that

$$
M_{11}=M_{22}
$$

From (B-35) follows that $\Gamma_{11}\left(\Gamma_{22}\right)$ is total decay width of $K^{0}\left(\bar{K}^{0}\right)$. Taking into account the $C P T$ invariance we have

$$
\Gamma_{11}=\Gamma_{22}
$$

Thus, if the CPT invariance holds we have

$$
\mathcal{H}_{11}=\mathcal{H}_{22}
$$

In the case of the $C P$ invariance we have

$$
\mathcal{H}_{11}=\mathcal{H}_{22}
$$

and

$$
\mathcal{H}_{12}=\mathcal{H}_{21} \text {. }
$$

If the relation (B-39) is violated this means that $C P T$ and $C P$ are violated. The violation of the relation $(\bar{B}-41)$ is a signature of the $C P$ violation.

The relation (B-41) was obtained under the assumption that the arbitrary phases of the states are chosen in such a way that $\left|\bar{K}^{0}\right\rangle=C P\left|K^{0}\right\rangle$. If we change the basic states and instead of $\left|K^{0}\right\rangle$ and $\left|\bar{K}^{0}\right\rangle$ will use $\left|K^{0}\right\rangle^{\prime}=e^{i \alpha}\left|K^{0}\right\rangle$ and $\left|\bar{K}^{0}\right\rangle^{\prime}=e^{-i \alpha}\left|\bar{K}^{0}\right\rangle$ we will have

$$
\mathcal{H}_{12}^{\prime}=e^{-4 i \alpha} \mathcal{H}_{21}^{\prime}
$$

Thus, there is no any relations between the phases of the non diagonal elements of the matrix $\mathcal{H}$ in the case of the $C P$ invariance. Only the violation of the relation

$$
\left|\mathcal{H}_{12}\right|=\left|\mathcal{H}_{21}\right|
$$

is a signature of the $C P$ violation.

Let us notice that in the case of $T$ invariance we have

$$
\left|\mathcal{H}_{12}\right|=\left|\mathcal{H}_{21}\right| \text {. }
$$

It is obvious that all relations we derived here are also valid for $B_{d, s}^{0}-\bar{B}_{d, s}^{0}$, $D^{0}-\bar{D}^{0}$ and other systems. 


\section{References}

[1] C.S. Wu et al, Phys. Rev. 105 (1957) 1413.

[2] L. Landau, Nucl. Phys. 3, 127 (1957).

[3] T.D. Lee and C.N. Yang, Phys. Rev. 105, 1671 (1957).

[4] A. Salam, Nuovo Cimento 5 (1957) 299.

[5] M. Goldhaber, L. Grodzins and A.W. Sunyar, Phys. Rev. 109, 1015 (1958).

[6] J.H. Christenson, J.W. Cronin, V. L. Fitch and R. Turlay, Phys. Rev. Lett. 13 (1964) 138.

[7] L. Wolfenstein, Phys. Rev. Lett. 33 (1964) 562.

[8] J.R. Batley et al (NA48 Collaboration) Phys. Lett. B544 (2002) 97.

[9] A. Alavi-Harati et al (KTeV Collaboration), Phys. Rev. D67 (2003) 012005.

[10] S.L. Glashow, Nucl. Phys. 22, 597 (1961).

[11] S. Weinberg, Phys. Rev. Lett. 19, 1264 (1967).

[12] A. Salam, Proc. of the $8^{\text {th }}$ Nobel Symposium on Elementary Particle Theory, Relativistic Groups and Analyticity, edited by N. Svartholm, 1969.

[13] A. J. Buras, Proceedings of the Intern. School on Subnuclear Physics, ed. A. Zichichi (World Scientific 2000) pp. 200-237; hep-ph /0101336.

[14] M. Kobayashi and T. Maskawa, Prog. Theor. Phys. 49, 652 (1973).

[15] Proc. of CP Violation, edited by C. Jarlskog, Advanced Series in High Energy Physics Vol. 3, p. 3, World Scientific, Singapore, 1989.

[16] The BaBar Physics Book, eds. P.Harrison and H. Quinn (1998) SLACR-0504.

[17] Heavy Flavors II, eds. A. Buras and M. Lindner, World Scientific. 
[18] G.C. Branco, L.Lavoura and J.P. Silva, "CP Violation" Clarendon PressOxford (1999)

[19] I.I. Bigi and A.I. Sanda, "CP Violation" Cambridge Monographs on Particle Physics, Nuclear Physics and Cosmology, Cambridge University Press, Cambridge (2000)

[20] B-Physics at the Tevatron:run II and beyond, hep-ph/0201071.

[21] K. Kleinknecht, Springer Tracts Mod. Phys. 195 (2003) 1.

[22] M. Battaglia et al, The CKM matrix and the unitary triangle (CERN, Geneva, 2003), hep-ph/0304132.

[23] J.P. Silva, Phenomenological aspects of $C P$ violation, hep-ph/0410351.

[24] Y. Nir, CP violation in meson decays, hep-ph/0510413.

[25] R. Fleisher, Flavor Physics and $C P$ violation, hep-ph/0608019.

[26] A. J. Buras, Flavor Physics and $C P$ violation, hep-ph/0505175.

[27] J. A. Hewett et al, The Discovery Potential of a Super B Factory, hep-ph/0503261.

[28] S. Weinberg, The Quantum Theory of Fields v.I Foundations, Cambridge University Press.

[29] S.M. Bilenky, Introduction to Feynman Diagrams and Electroweak Interaction Physics, Editions Frontiers, 1964.

[30] N. Cabibbo, Phys. Lett. 10 (1963) 531.

[31] C. Jarlskog, Phys. Rev. Lett. 55 (1985) 1039; C. Jarlskog, Z. Phys. C29 (1985) 491. C. Jarlskog and R.Stora, Phys. Lett. B208 (1988) 268.

[32] W. M. Yao et al (Particle Data Group ), J. Phys.G 33 (2006) 1.

[33] A. Ceccuci, Z. Ligeti and Y. Sakai "The CKM quark-mixing matrix" in W. M, Yao et al (Particle Data Group), J. Phys.G 33 (2006) 1.

[34] J. C. Hardy and I. S. Towner, Phys. Rev. Lett. 94 (2005) 092502. 
[35] G. Savard et al, Phys. Rev. Lett. 95 (2005) 102501.

[36] D. Pocanic et al, Phys. Rev. Lett. 93 (2004) 181803.

[37] E. Blucher and W. J. Marciano, " $V_{u d}, V_{u s}$. The Cabibbo angle and CKM unitarity" in W. M, Yao et al (Particle Data Group ), J. Phys.G 33 (2006) 1.

[38] T.Alexopoulos et al (KTeV collaboration), Phys. Rev. Lett. 93 (2004) 181802; T.Alexopoulos et al Phys. Rev. D70 (2004) 092006.

[39] F. Ambrosino et al (KLOE collaboration), Phys. Lett. B632 (2006) 43.

[40] A. Lai et al (NA48 collaboration), Phys. Lett. B602 (2004) 41.

[41] H. Leutwyler and M. Roos, Z. Phys, C25 (1984) 91.

[42] C. Bernard et al, PoS LAT2005 (2005) 025; hep-lat/0509137.

[43] N. Cabibbo, E. S. Swallow and R. Winston, Phys. Rev. Lett. 92 (2004) 251803.

[44] E. Gamiz et al, Phys. Rev. Lett. 94 (2005) 011803.

[45] C. Aubin et al, Phys. Rev. Lett. 94 (2005) 011601.

[46] M. Artuso, Int.J.Mod.Phys. A21 (2006) 1697; hep-ex/0510052.

[47] P. Abren et al, Phys. Lett. B439 (1998) 209.

[48] I.I.Y.Bigi et al, Phys. Rev. Lett. 71 (1993) 496.

[49] A.V. Manohar and M.B. Weise, Phys. Rev. D49 (1994) 1310.

[50] B. Kowalewski and T. Mannel, "Determination of $V_{c b}$ and $V_{u b}$ " in W. M, Yao et al (Particle Data Group ). J. Phys.G 33 (2006) 1.

[51] N. Isgur and M.B. Wise, Phys. Lett. B232 (1989) 113; Phys. Lett. B237 (1990) 527.

[52] M.A. Shifman and M.B. Voloshin, Sov. J. Nucl. Phys. 47 (1988) 511 (Yad. Phys. 47 (1988) 801.

[53] M. Okamoto et al, Nucl. Phys. (Proc. Supp.) B140 (2005) 461. 
[54] J. Shigemitsu et al, Nucl. Phys. (Proc. Supp.) B140 (2005) 464.

[55] E. Barbero et al (Heavy Flavor Areraging Group), hep-ex/0603003.

[56] A. Gray et al, Phys. Rev. Lett. 95 (2005) 212001.

[57] S. Aoki et al, Phys. Rev. Lett. 91 (2003) 212001.

[58] M. Okamoto et al, PoS LAT2005 (2005) 013.

[59] A. Abulencia et al (CDF collaboration), Phys.Rev.Lett.97 (2006) 062003; hep-ex/0606027.

[60] D. Acosta et al (CDF collaboration), Phys. Rev. Lett. 95 (2005) 102002.

[61] V.M. Abazov et al (D0 collaboration), Phys.Lett.B639 (2006) 616; hep-ex/0603002.

[62] L. Wolfenstein, Phys. Rev. Lett. 51 (1983) 1945.

[63] A. Buras, E.M. Lautenbacher and G. Ostermaier, Phys. Rev. D50 (1994) 3433.

[64] L. Wolfenstein and T.G. Trippe " $C P$ vilation in $K_{L}$ decays" in W. M, Yao et al (Particle Data Group), J. Phys.G 33 (2006) 1.

[65] G. Calangelo, J. Gasser and H. Leutwyler, Nucl. Phys.B603 (2001) 125.

[66] U. Nierste, hep-ph/0612310.

[67] A.B. Carter and A.I. Sanda, Phys. Rev. Lett. 45 (1980) 952; Phys. Rev. D23 (1981) 1567; I.I. Bigi and A.I. Sandra, Nucl. Phys. 193 (1981)85.

[68] B. Aubert et al (BaBar Collaboration), Phys.Rev. Lett. D 66 (2002) 071102 .

[69] K. Abe et al (Belle Collaboration), Phys.Rev. 89 (2002) 201802.

[70] B. Aubert et al (BaBar Collaboration), hep-ex/0607107.

[71] K. Abe et al (Belle Collaboration), hep-ex/0608039.

[72] M. Bona et al (UTfit Collaboration), hep-ph/0501199. 
[73] M. Bona et al (UTfit Collaboration), JHEP 0610 (2006) 081; hep-ph/0606167.

[74] J. Charles et al (CKMfitter group), Eur. Phys. J. 41 (2005)1; hep-ph/0406184; hep-ph/0606046.

[75] E. Barberio et al (Heavy Flavor Averaging Group), hep-ex/0603003.

[76] M. Rama, Proceedings of XXI Rencontres de Physique de la Vallee d'Aoste, March 4-10, 2007.

[77] C.S. Wu et al, Phys. Rev. 105 (1957) 1413.

[78] R.P.Feynman and M.Gell-Mann, Phys. Rev.109 (1958) 193.

[79] E.C.G. Sudarshun and R. Marshak, Phys. Rev. 109 (1958) 1860.

[80] Y. Ashie et al., (Super-Kamiokande Collaboration), Phys. Rev. Lett. 93 (2004) 101801; Phys. Rev. D71 (2005) 11205.

[81] SNO Collaboration, Phys. Rev. Lett. 87 (2001) 071301; 89 (2002) $011301 ; 89$ (2002) 011302; 92 (2004) 181301.

[82] T.Araki et al. (KamLAND Collaboration), T.Araki et al., Phys. Rev. Lett. 94 (2005) 081801; hep-ex/0406035.

[83] B. T. Cleveland et al., Astrophys. J. 496 (1998) 505.

[84] W. Hampel et al. (GALLEX Collaboration), Phys. Lett. B 447 (1999) 127 ; GNO Collaboration, M. Altmann et al., Phys. Lett. B 490 (2000) 16 ; Nucl. Phys. Proc. Suppl. 91 (2001) 44; Phys. Lett. B 616 (2005) 174 .

[85] J. N. Abdurashitov et al. (SAGE Collaboration), Phys. Rev. C 60 (1999) 055801; Nucl. Phys. Proc. Suppl. 110 (2002) 315; Nucl. Phys. Proc. Suppl. 118 (2003) 39.

[86] S. Fukuda et al. (Super-Kamiokande Collaboration), Phys. Rev. Lett. 86 (2001) 5651.

[87] M.H. Ahn et al. (K2K Collaboration), Phys. Rev. Lett. 90 (2003) 041801. 
[88] D.G. Michael et al. ( MINOS Collaboration), Phys.Rev.Lett. 97 (2006) 191801; arXiv:hep-ex/0607088.

[89] S. Bilenky and C. Giunti, Int.J.Mod.Phys. A16 (2001) 3931; hep-ph/0102320.

[90] S.M. Bilenky and M.D. Mateev, Phys.Part.Nucl.38 (2007) 117; hep-ph/0604044.

[91] B. Pontecorvo, J. Exptl. Theoret. Phys. 33 (1957) 549. [Sov. Phys. JETP 6 (1958) 429 ]; J. Exptl. Theoret. Phys. 34 (1958) 247 [Sov. Phys. JETP 7 (1958) 172 ].

[92] Z. Maki, M. Nakagawa and S. Sakata, Prog. Theor. Phys. 28 (1962) 870.

[93] S.M. Bilenky and S.T. Petcov, Rev. Mod. Phys. 59, 671 (1987).

[94] S.M. Bilenky, C. Giunti, and W. Grimus. Prog. Part. Nucl. Phys. 43 (1999) 1.

[95] S.M. Bilenky, N.P. Nedelcheva and S.T. Petcov, Nucl. Phys.B247(1984) 61; B. Kayser, Phys. Rev.D30 (1984) 1023.

[96] S.M. Bilenky, J. Hosek and S.T. Petcov, Phys. Lett.B94 (1980) 495.

[97] V.Weisskopf and E. Wigner, Z. fur Physik, 63 (1930) 54, Z. fur Physik, 65 (1930) 18. 